\title{
Fuselage Excitation During Cruise Flight Conditions: Measurement and Prediction of Pressure Point Spectra
}

\author{
Alexander Klabes, Christina Appel ${ }^{\dagger}$ and Michaela Herr ${ }^{\dagger}$ \\ German Aerospace Center (DLR), D-38108 Braunschweig, Germany \\ Mohamed Bouhaj ${ }^{\ddagger}$ \\ Airbus Operations GmbH, D-21129 Hamburg, Germany
}

\begin{abstract}
In the last fifty years many semi-empirical models to predict surface pressure fluctuations beneath turbulent boundary layers (TBL) have been developed for a large variety of test conditions. Nowadays, the relevance of the TBL as a source of cabin interior noise is steadily increasing, due to quieter aircraft engines. The possibility of predicting surface pressure auto-spectra with the various publicly available semi-empirical models at several positions on the fuselage of DLR's Advanced Technology Research Aircraft (ATRA) is investigated. A large validation database was used, involving in-flight measurements at different flight levels (FL) and Mach numbers, applying two different sensor types. Predictions were performed based on semi-empirically estimated TBL parameters (partly included in the different models) and, additionally, based on TBL properties that were extracted from CFD simulations. This procedure served to identify different sources of error in the prediction. Overall, it is shown that today's models provide a large ( $>10 \mathrm{~dB})$ scatter among the predicted spectra. Even the most suitable approaches are not generally applicable to all relevant positions at the fuselage. Particularly in regions with strong pressure gradients and high turbulence kinetic energy measured auto-spectra cannot be reproduced with sufficient accuracy. This indicates the need for more universally applicable CFD- and CAA-based surface pressure prediction methods.
\end{abstract}

\section{Nomenclature}

Latin nomenclature

$b$

$d$

$f$

$f_{0} \quad$ Characteristic frequency $[\mathrm{Hz}]$

$k \quad$ Specific turbulence kinetic energy $\left[\mathrm{m}^{2} / \mathrm{s}^{2}\right]$

$\overline{{p^{\prime}}^{2}} \quad$ Mean square pressure $\left[\mathrm{Pa}^{2}\right]$

$q \quad$ Dynamic pressure $[\mathrm{Pa}]$

$r \quad$ TBL model empirical constant, EfImTsov [-]

$u_{i}^{\prime} \quad$ Turbulent fluctuations $[\mathrm{m} / \mathrm{s}]$

$x \quad$ Running length [m]

$C_{M, T} \quad$ TBL model empirical constants, Chase [-]

$C_{1} \quad$ TBL model empirical constants, RACKL \& Weston [-]

FL Flight level [100 ft]

$G_{a-h} \quad$ TBL model empirical constants, Goody [-]

\footnotetext{
*PhD candidate, Institute of Aerodynamics and Flow Technology - Technical Acoustics, alexander.klabes@dlr.de

${ }^{\dagger}$ Research Scientist, Institute of Aerodynamics and Flow Technology - Technical Acoustics

${ }^{\ddagger}$ Acoustic Engineer, Interior \& Near Field Noise, Acoustics \& Environment - EPA5
} 


$\begin{array}{ll}M a & \text { Mach number [-] } \\ P_{t o} & \text { Total pressure [Pa] } \\ R_{T} & \text { Ratio of the outer- to inner-layer timescale [-] } \\ R e & \text { Reynolds number [-] } \\ R e_{\tau} & \text { Shear stress Reynolds number [-] } \\ R e_{\theta} & \text { Momentum Reynolds number [-] } \\ S h & \text { Strouhal number [-] } \\ T & \left.\text { Temperature }{ }^{\circ}\right] \\ U_{c} & \text { Convective }(\text { or phase) velocity of the turbulence }[\mathrm{m} / \mathrm{s}] \\ U_{e} & \text { Velocity at the boundary-layer edge }[\mathrm{m} / \mathrm{s}] \\ U_{\tau} & \text { Friction velocity }[\mathrm{m} / \mathrm{s}] \\ U_{\infty} & \text { Freestream velocity }[\mathrm{m} / \mathrm{s}]\end{array}$

Greek nomenclature

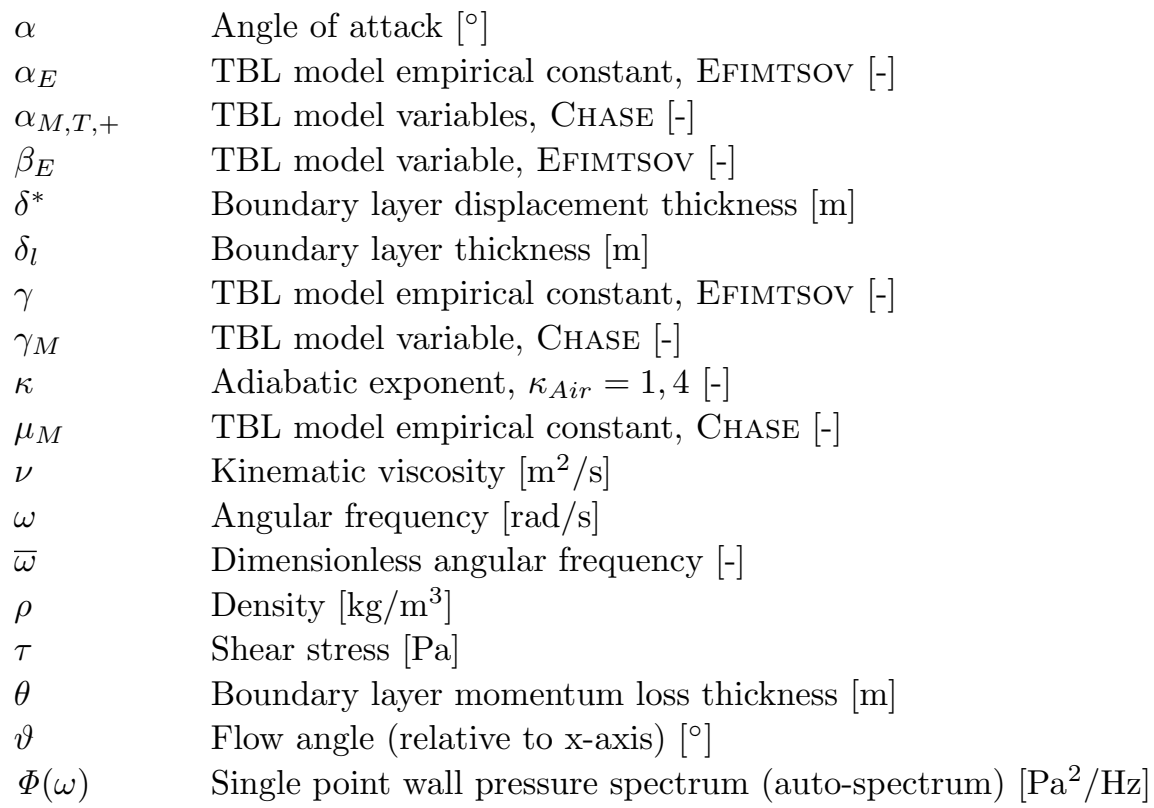

List of indices

$\begin{array}{ll}\infty & \text { Freestream } \\ 0 & \text { Reference condition } \\ w & \text { Wall }\end{array}$

General abbreviations

ATRA Advanced Technology Research Aircraft

CAA Computational aeroacoustics

CFD Computational fluid dynamics

DLR German Aerospace Center

FL Flight level [100 ft]

FT Flight test

HTP Horizontal tail plane

ISA International Standard Atmosphere

NASA National Aeronautics and Space Administration 


$\begin{array}{ll}\text { RSM } & \text { Reynolds-stress model } \\ \text { SAO } & \text { Spalart-Allmaras original turbulence model } \\ \text { SPL } & \text { Sound pressure level } \\ \text { TBL } & \text { Turbulent boundary layer } \\ \text { TKE } & \text { Turbulence kinetic energy } \\ \text { TsAGI } & \text { Central Aerohydrodynamic Institute in Moscow, Russia } \\ \text { TU } & \text { Tupolew } \\ \text { VTP } & \text { Vertical tail plane }\end{array}$

\section{Introduction}

PEOple will spend more and more time on planes, whether for private or business purposes, and their comfort expectance will successively grow. Among other demands on passenger comfort, aircraft cabin noise should be as low as possible. One of the main cabin noise contributors is the turbulent boundary layer (TBL), causing fluctuating pressures on the fuselage. Via complex transfer paths through the fuselage structure into the cabin these pressure fluctuations excite structural vibrations and noise. TBL-induced surface pressures are currently modelled based on variant semi-empirical approaches, which include the description of the local pressure fluctuations (auto-spectra) as well as the space- and time-dependent field behaviour (cross-spectra). According to Miller, ${ }^{1}$ available prediction methods can be classified into three categories, sorted by complexity of the used field statistics and prediction output, i.e. models which are mainly described by

1. the overall mean square pressure as a direct measure of the energy due to the pressure fluctuations beneath the TBL,

2. the single point wall pressure spectrum (auto-spectrum) sorting this energy into frequencies, or

3. the wavenumber-frequency spectrum, further sorting the energy into wavenumbers, i. e. providing correlation information in time and space.

Test conditions while deriving available models ranged from subsonic to supersonic flows and included measurements from laboratory to full scale, i. e. it is expected that the applicability of the respective models might be limited to groups of similar test cases. In this paper it is investigated how reliable the variant predictions of the single-point spectrum are compared to an independent measurement data base. Here, surface pressure measurement data from several flight test campaigns with DLR's A320 Advanced Technology Research Aircraft (ATRA) are used for this evaluation. Flight tests are in parts documented by SpeHR et al. ${ }^{2}$ Forerunner work on this database by HAXTER and SPEHR ${ }^{3-5}$ focused on the cross-spectral analysis in selected smaller regions of the fuselage, whereas the current contribution is dedicated to the spectral characteristics (like spectral peak and shape) of the point spectra at arbitrary fuselage positions. Corresponding cabin noise measurements results were reported by $\mathrm{Hu}$ et al.,,${ }^{6,7}$ to complete the picture.

The structure of the current report is as follows: Section II provides a short overview of the semi-empirical models that have been revisited within this study. In section III the used measurement setup is documented, while the flight test data analysis is summarized in section IV. Here, the effects of parameters like flight Mach number and flight level on the measured spectra are shown. To gain comprehensive knowledge of the local TBL characteristics (like local boundary layer thickness, flow velocity, etc.), CFD simulations of the ATRA flow field during cruise were conducted. The results are provided in section V. Finally, section VI concludes with the comparison of the flight test data and the predictions. This section contains a sensitivity analysis with regard to the quality of TBL input parameters used for the predictions. On the one hand the semi-empirical models were fed with estimated TBL parameters based on flat plate theory, as it is current practice in some of the approaches, whereas on the other hand extracted parameters from the CFD analysis were applied instead. 


\section{Semi-empirical models for the prediction of point spectra}

This section contains a brief description of nine semi-empirical models for the prediction of surface pressure auto-spectra, without claiming to be exhaustive.

\section{A. Robertson (1971)}

The model by ROBERTSON is based on the work of LOWSON ${ }^{8}$ and constitutes an improvement at low and high Strouhal numbers, published in 1971. ROBERTSON defines the auto-spectrum as follows, by comparing the Lowson model with other data, and in particular, with measurements at supersonic speeds by NASAAmes: ${ }^{9}$

$$
\Phi(\omega)=\frac{\overline{p^{\prime 2}}}{\omega_{0}\left[1+\left(\frac{\omega}{\omega_{0}}\right)^{0.9}\right]^{2}},
$$

with the characteristic frequency

$$
\omega_{0}=0.5 \frac{U_{\infty}}{\delta^{*}} .
$$

Where $\omega$ is the angular frequency, $U_{\infty}$ the free stream velocity and $\delta^{*}$ the local boundary layer displacement thickness. Furthermore, the mean square pressure fluctuation is defined as

$$
\overline{{p^{\prime}}^{2}}=\left(\frac{0.006 q_{\infty}}{1.0+0.14 M a_{\infty}^{2}}\right)^{2},
$$

with the dynamic pressure $q_{\infty}=\frac{1}{2} \rho U_{\infty}^{2}$. This formulation of the mean square pressure fluctuation is introduced by Lowson. ${ }^{8}$ Good agreement of calculated values with experimental results is shown in the Mach number range from $M a_{\infty}=0.6$ up to $M a_{\infty}=3.0 .^{9}$

\section{B. Cockburn \& Robertson (1974)}

For attached turbulent flow, COCKBURn \& ROBERTSON stated the formula which was previously derived by Robertson (Eq. (1)). Cockburn \& Robertson conducted tests on a $15^{\circ}$ cone-cylinder payload shroud at three Mach numbers $\left(M a_{\infty, 1}=0.7, M a_{\infty, 2}=0.8, M a_{\infty, 3}=2.0\right) .{ }^{10}$ Their presented results were derived for an Atlas-Agena launch vehicle, fitted with a standard payload shroud comprising fiberglass skin and aluminium ring-frame stiffeners. The model is $1.676 \mathrm{~m}$ in diameter and $5.791 \mathrm{~m}$ long, wherein the cylindrical section has a length of $3.302 \mathrm{~m}$. Rewriting Eq. (1) as in the 1974 paper of CockBurn \& RoBertson yields

$$
\Phi(f)=\frac{\overline{p^{\prime 2}}}{f_{0}\left[1+\left(\frac{f}{f_{0}}\right)^{0.9}\right]^{2}} .
$$

At first glance this model seems to be equal to the Robertson model, but CockBurn \& Robertson use a modified estimate of the characteristic frequency

$$
f_{0}=0.346 \frac{U_{\infty}}{\delta_{l}} .
$$

The estimate of the characteristic frequency used here is based on the local TBL thickness $\delta_{l}$ instead of $\delta^{*}$. As the mean square pressure fluctuation formulation, Eq. (3) is used.

\section{Chase $(1980 / 1987)$}

According to HWANG ${ }^{11}$ the CHASE model for the single point wall pressure spectrum is

$$
\Phi(\omega)=\frac{\rho^{2} U_{\tau}^{4}}{\omega}\left[\frac{\alpha_{+} \gamma_{M}}{\alpha_{M}{ }^{3}}\left(1+\mu_{M}^{2} \alpha_{M}^{2}\right)+\frac{3 \pi C_{T}}{\alpha_{T}}\left(1+\alpha_{T}{ }^{-2}\right)\right] .
$$


A first formula for the wavenumber-frequency spectrum was published by CHASE in $1980 .{ }^{12}$ CHASE improved his model further and published the latest version of the wavenumber-frequency spectrum in $1987,{ }^{13}$ which is the starting point for Eq. (6). Equation (6) is obtained by integrating the wavenumber-frequency spectrum over the wavevector plane.

The constants and variables in Eq. (6) are defined as $\alpha_{M}=\sqrt{1+\left(\frac{b \omega \delta_{l}}{U_{c}}\right)^{-2}}, \alpha_{T}=\sqrt{1+\left(\frac{b \omega \delta_{l}}{U_{c}}\right)^{-2}}$, $C_{M}=0.1553, C_{T}=0.00476, b=0.75, \mu_{M}=0.176, \alpha_{+}=2 \pi\left(C_{M}+C_{T}\right), \gamma_{M}=\frac{C_{M}}{C_{M}+C_{T}}$.

Furthermore, $\mathrm{CHASE}^{13}$ defined the friction velocity as $U_{\tau}=\sqrt{\tau_{w} / \rho}$. In the equations for $\alpha_{M}$ and $\alpha_{T}$ one parameter is the convective velocity $\left(U_{c}\right)$ of the turbulence in the turbulent boundary layer. According to CHASE $^{12,13}$ this value is between $U_{c}=0.65 \cdot U_{\infty}$ and $U_{c}=0.75 \cdot U_{\infty}$. This value can be determined more exactly by using the phase velocity of the cross-spectrum between two sensors aligned with the flow direction. Following that approach, ratios between convective and free stream velocity of about $\frac{U_{c}}{U_{\infty}}=0.75-0.80$ were measured frequency dependent for in-flight data. ${ }^{14}$

\section{Efimtsov (1982/1984)}

The Efimtsov 1 model $^{15}$ is dependent on Mach number (Ma), Reynolds number (Re) and Strouhal number (Sh), in this case defined as $S h=\frac{\omega \delta_{l}}{U_{\tau}} \cdot{ }^{15}$ The model is based on flight test data in the range of Mach numbers $\mathrm{Ma}=0.41 \ldots 2.1$, and Reynolds numbers of $\mathrm{Re}=0.5 \cdot 10^{8} \ldots 4.85 \cdot 10^{8}$. The pressure fluctuations were measured at various positions on an aircraft fuselage, where the boundary layer was considered fully developed with zero pressure gradient. EFIMTSOv's single point wall pressure spectrum is given by:

$$
\Phi(\omega)=\frac{0.01 \tau_{w}^{2} \delta_{l}}{U_{\tau}\left[1.0+0.02\left(\frac{\omega \delta_{l}}{U_{\tau}}\right)^{\frac{2}{3}}\right]} .
$$

The Efimtsov 2 model $^{16}$ is a further development of the Efimtsov 1 model (Eq. (7)), using additional data from low and high speed TsAGI (Central Aerohydrodynamic Institute in Moscow, Russia) wind tunnels. The measurements span Mach numbers from $\mathrm{Ma}=0.015 \ldots 4.0$ and Reynolds numbers from $\mathrm{Re}=6 \cdot 10^{2} \ldots 1.5$. $10^{5} .{ }^{16}$ According to EFIMTSOV ${ }^{16}$ the single point wall pressure spectrum model from 1984 is

$$
\Phi(\omega)=\frac{\alpha_{E} U_{\tau}^{3} \rho^{2} \delta \beta_{E}}{\left(1+8 \alpha_{E}{ }^{3} S h^{2}\right)^{\frac{1}{3}}+\alpha_{E} \beta_{E} R e_{\tau}\left(\frac{S h}{R e_{\tau}},\right)^{\frac{10}{3}}},
$$

with $R e_{\tau}=\frac{\delta_{l} U_{\tau}}{\nu_{w}}, R e_{\tau 0}=3000, S h=\frac{\omega \delta_{l}}{U_{\tau}}, \beta_{E}=\left[1+\left(\frac{R e_{\tau 0}}{R e_{\tau}}\right)^{3}\right]^{\frac{1}{3}}, \alpha_{E}=0.01, \nu_{w}=\nu \frac{\rho}{\rho_{w}}\left(\frac{T_{w}}{T_{\infty}}\right)^{\gamma}$, $\gamma=0.905, T_{w}=T_{\infty}\left(1+r \frac{\kappa-1}{2} M a^{2}\right), r=0.89, \kappa=1.4, \rho_{w}=\rho \frac{T_{\infty}}{T_{w}}$.

According to RACKL \& WESTON ${ }^{17}$ also flight measurements on a TU144 and a russian twin engined supersonic military aircraft (TU-22) are taken into account for this model. RACKL states that the sensor positions appear to be similar to those used on a TU144 by NASA-Boeing.

\section{E. Chase-Howe (1998)}

The single point wall pressure spectrum is given by the CHASE - Howe model ${ }^{1}$ as

$$
\Phi(\omega)=\frac{2\left(\frac{\delta^{*}}{U_{\infty}}\right)^{3}\left(\tau_{w} \omega\right)^{2}}{\left[\left(\frac{\omega \delta^{*}}{U_{\infty}}\right)^{2}+0.0144\right]^{\frac{3}{2}}} .
$$

The Chase - Howe model was presented by Howe ${ }^{18}$ and is based on the CHASE model. It is much simpler than the model proposed by CHASE and the first time refered to as the CHASE - HowE model by Goody. ${ }^{19}$ 
Compared to the Chase model, the modified CHASE - HOwE model takes not as many TBL variables into account, which is evidence for the lower degree of complexity of the CHASE - HowE model.

\section{F. Smol'yakov (2000)}

SMOL'YAKOV proposed a new model using different scaling variables for different frequency regions. He discribed three regions in the spectrum, a low frequency, an universal and a high frequency region. The model proposed by Smol'yakov is analogue to the three mentioned regions devided in three formulae. ${ }^{20}$ The SMOL'YAKOV model is based on a thorough analysis of his theoretical model of the wavenumber-frequency spectrum and a diverse group of data reportet in the literature. ${ }^{11}$

1. Low frequency region at $\bar{\omega}<\bar{\omega}_{0}$

$$
\Phi(\omega)=1.49 \cdot 10^{-5} \cdot \operatorname{Re}_{\theta}^{2.74} \bar{\omega}^{2}\left(1-0.117 \operatorname{Re}_{\theta}{ }^{0.44} \bar{\omega}^{1 / 2}\right) \cdot \frac{\tau_{w}^{2} \nu}{U_{\tau}^{2}}
$$

2. Universal frequency region at $\bar{\omega}_{0}<\bar{\omega}<0.2$

$$
\Phi(\omega)=2.75 \bar{\omega}^{-1.11}\left(1-0.82 \exp \left[-0.51\left(\frac{\bar{\omega}}{\bar{\omega}_{0}}-1\right)\right]\right) \cdot \frac{\tau_{w}^{2} \nu}{U_{\tau}^{2}}
$$

3. High frequency region at $\bar{\omega}>0.2$

$$
\begin{aligned}
\Phi(\omega)= & \left(38.9 e^{-8.35 \bar{\omega}}+18.6 e^{-3.58 \bar{\omega}}+0.31 e^{-2.14 \bar{\omega}}\right) \\
& \cdot\left(1-0.82 \exp \left[-0.51\left(\frac{\bar{\omega}}{\bar{\omega}_{0}}-1\right)\right]\right) \cdot \frac{\tau_{w}^{2} \nu}{U_{\tau}^{2}}
\end{aligned}
$$

Equations (10) - (12) are valid at $\operatorname{Re}_{\theta}>10^{3}\left(\operatorname{Re}>5 \cdot 10^{5}\right)$. Furthermore $\bar{\omega}$ and $\bar{\omega}_{0}$ are defined by $\bar{\omega}=\omega \nu / U_{\tau}^{2}$ respectively $\bar{\omega}_{0}=49.35 R e_{\theta}^{-0.88}$, these values are dimensionless frequencies.

According to reference 20, the first factors in the formulae (Eq. (10) - (12)) describe the main laws governing the behaviour of the spectra in the low frequency, universal, and high frequency ranges, respectively. The second factor (in parentheses) provide a smooth matching of the levels in the regions between these ranges. The used momentum Reynolds number $R e_{\theta}$ is defined as

$$
R e_{\theta}=\frac{U_{\infty} \cdot \theta}{\nu},
$$

with the boundary layer momentum loss thickness $\theta$.

\section{G. Goody (2004)}

The single point wall pressure spectrum of the GooDy model ${ }^{19}$ is given by

$$
\frac{\Phi(\omega) U_{e}}{\tau_{w}^{2} \delta_{l}}=\frac{G_{a}\left(\frac{\omega \delta_{l}}{U_{e}}\right)^{G_{b}}}{\left[\left(\frac{\omega \delta_{l}}{U_{e}}\right)^{G_{c}}+G_{d}\right]^{G_{e}}+\left[\left(G_{f} R_{T}^{G_{g}}\right)\left(\frac{\omega \delta_{l}}{U_{e}}\right)\right]^{G_{h}}},
$$

with $G_{a-h}=3.0,2.0,0.75,0.5,3.7,1.1,-0.57,7.0$ and $R_{T}=\frac{U_{\tau}^{2} \delta_{l}}{U_{e} \nu}$ is the ratio of the outer-layer-toinner-layer timescale, which can better be seen after rearranging $\left(R_{T}=\frac{\delta_{l}}{U_{e}} / \frac{\nu}{U_{\tau}^{2}}\right)$.

The Goody model offers a high degree of confidence when extrapolated to flows with a higher Reynolds number and zero pressure gradient. ${ }^{19}$ GoOdy used the CHASE - Howe model as a starting point for the development of his model. He reviewed the experimental surface pressure spectra of six research groups in his dissertation, ${ }^{21}$ that cover a large Reynolds number range: $1.4 \cdot 10^{3}<R e_{\theta}<2.34 \cdot 10^{4}, R e_{\theta}=\frac{U_{e} \theta}{\nu}$. This

Reynolds number span is representative for most laboratory flows, that is, wind tunnels and water tunnels $\left(\operatorname{Re}_{\theta} \sim 10^{3}-10^{4}\right)$. Most practical flows, like the flow over airplanes have a larger momentum Reynolds number $\left(\operatorname{Re}_{\theta} \sim 10^{5}-10^{6}\right) \cdot{ }^{19}$ 


\section{H. Rackl \& Weston (2005)}

RACKL and Westons model is an adjustment of the existing Efimtsov 2 model, they published their modification in 2005. ${ }^{17}$ Comparisons of measured flight test data from a TU 144LL with predictions from the EFIMTSOv 2 model showed two characteristics. The first was a broad band spectral peak around Strouhal number of $S h=0.6$, where Strouhal number is calculated as $S h=\frac{2 \pi f \delta^{*}}{U_{\infty}}$. As the second, data showed a slightly steeper roll-off at high frequencies (above $1 \mathrm{kHz}$ ) than the predicted roll-off. Speculations about the reasons for the observed deviations can be found in reference 17.

RACKL and WESTON used two adjustment functions, firstly

$$
C_{1} \exp \left(-\left(\ln (S h)-\ln \left(S h_{1}\right)\right)^{2}\right) ;\left(C_{1}=2.5\right),
$$

$S h_{1}=0.6$ is the reference value, where the spectral peak in the measurements appeared and secondly with the factor accounting for the steeper roll-off

$$
\frac{1}{4}\left(\tanh \left(\log \left(\frac{f}{1000}\right)\right)+1\right)[(M a-1.65) \log (f)] .
$$

In Eq. (16), since only the high frequency slope needs adjustment, a tanh-function centered at $1000 \mathrm{~Hz}$ is included. The second term in parentheses accounts for the overprediction of the EFIMTsov 2 model in the range below $\mathrm{Ma}=1.65$ and the underprediction for conditions above $\mathrm{Ma}=1.65$.

Finally it follows

$$
\begin{aligned}
\Phi(f)_{\text {adjusted }}= & \Phi(f)_{\text {predicted }} \\
& +2.5 \exp \left(-\left(\ln \left(\frac{2 \pi f \delta^{*}}{U_{\infty}}\right)-\ln (0.6)\right)^{2}\right) \\
& +\frac{1}{4}\left(\tanh \left(\log \left(\frac{f}{1000}\right)\right)+1\right)[(M a-1.65) \log (f)] .
\end{aligned}
$$

\section{Model Comparison}

In this section a plot with all semi-empirical TBL models is presented for FL 350 and Ma $=0.78$ at a running length of $x=12.5 \mathrm{~m}$. The results for this flight configuration can be seen in figure 1 .

Figure 1 shows that the models can be divided into two major groups. On the one hand the green and blue lines, describing the ROBERTSOn / COCKBURn \& ROBERTSOn, respectively the EFIMTsov / RACKL \& Weston models. These models are based on flight test and wind tunnel data at high Mach- and Reynolds numbers. Distinctive for this models is a large plateau in the low frequency range with a roll-off at higher frequencies.

On the other hand the Goody, Chase - Howe and Smol'yakov models represent the second group. This group obviously provides spectra with different shapes, especially in the low frequency range. The absolute values of these predictions increase with increasing frequency until a maximum in the mid frequency range is reached and then it is followed by a decreasing behaviour with increasing frequency.

The CHASE model is with regard to the shape, something in between this two mentioned groups. In the low frequency range a large plateau is characteristic, as it is for the ROBERTSON / COCKBURN \& ROBERTSON and the Efimtsov / RACKL \& Weston models. For higher frequencies the predicted spectrum peaks and afterwards it is characterised by a roll-off similar to the Goody, Chase - Howe and SMOL'yakov models. 


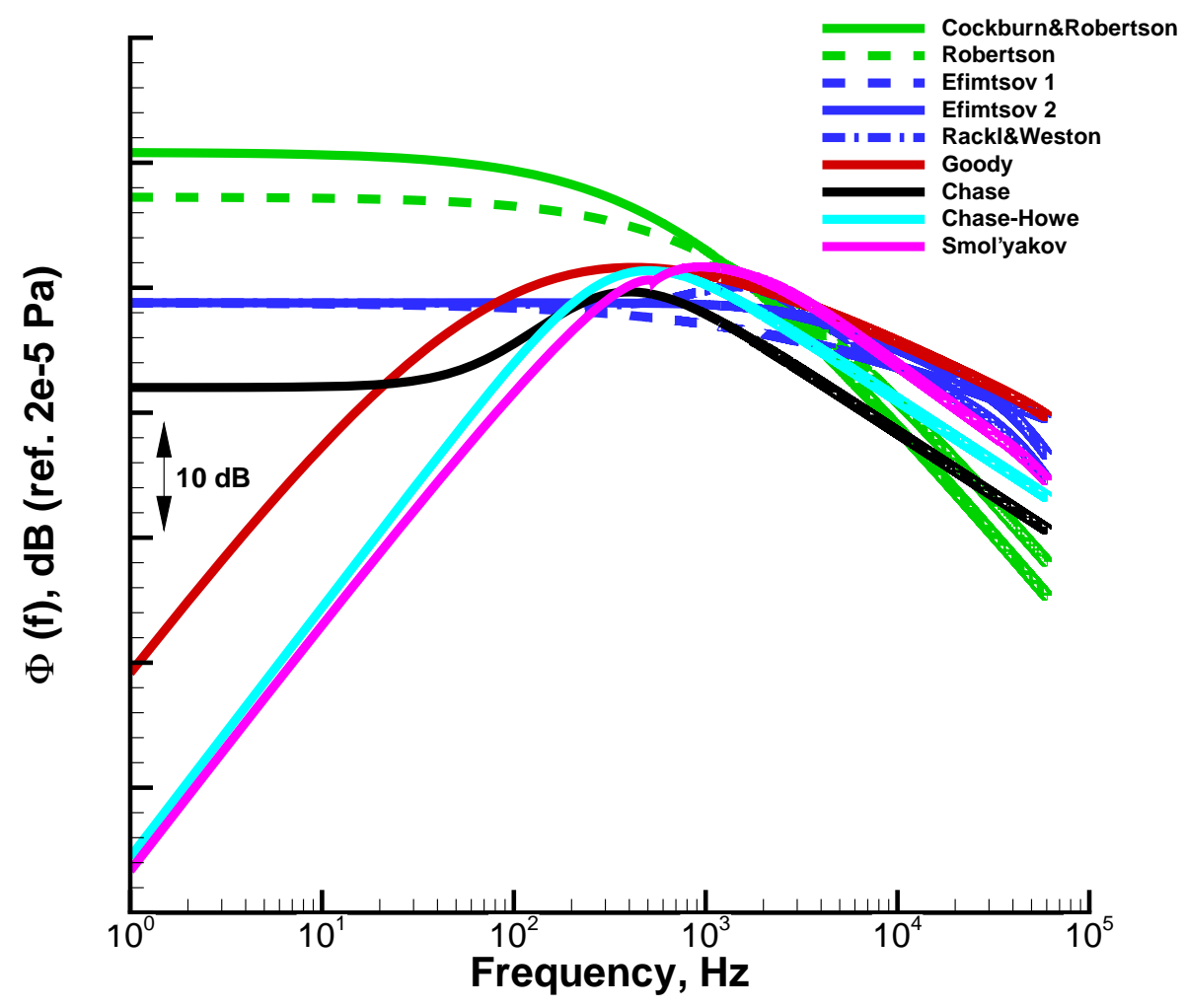

Figure 1. Comparison of predictions from all auto-spectra models; Ma $=0.78$, FL $350, x=12.5 \mathrm{~m}$ 


\section{Flight test measurement setup}

In this section an overview of the set of sensors installed on the ATRA fuselage is given. Data were acquired with two different sensor types at different positions on the fuselage. In figure 2, regions equipped with piezoresistive pressure transducers of type Kulite XCL-093 are coloured. The blue area represents the front measurement region, the red and green the middle and aft measurement region respectively. In each flight test three Kulite windows were installed next to each other, instead of standard passenger windows. Kulite sensors are installed behind a pinhole of $0.3 \mathrm{~mm}$ diameter. Detailed arrangement of the Kulite pressure transducers in each window can be seen in figure 3, with an array-fixed coordinate system, centered on the middle of window two. In the further analysis always the running length of the mid dummy window is specified.

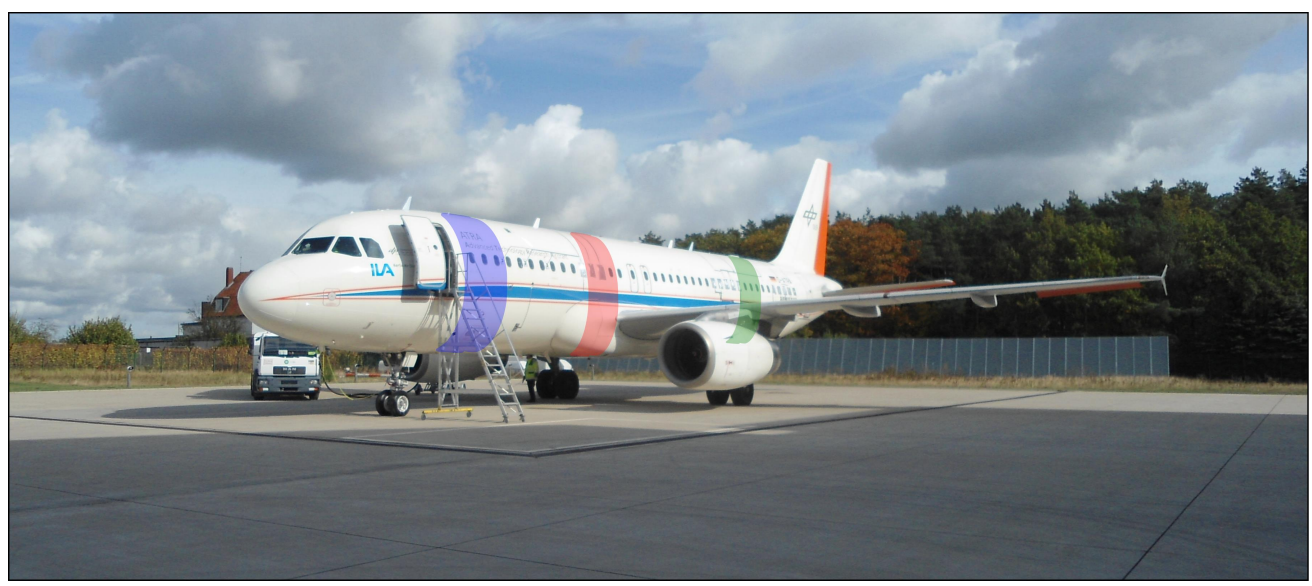

Figure 2. Kulite measurement region

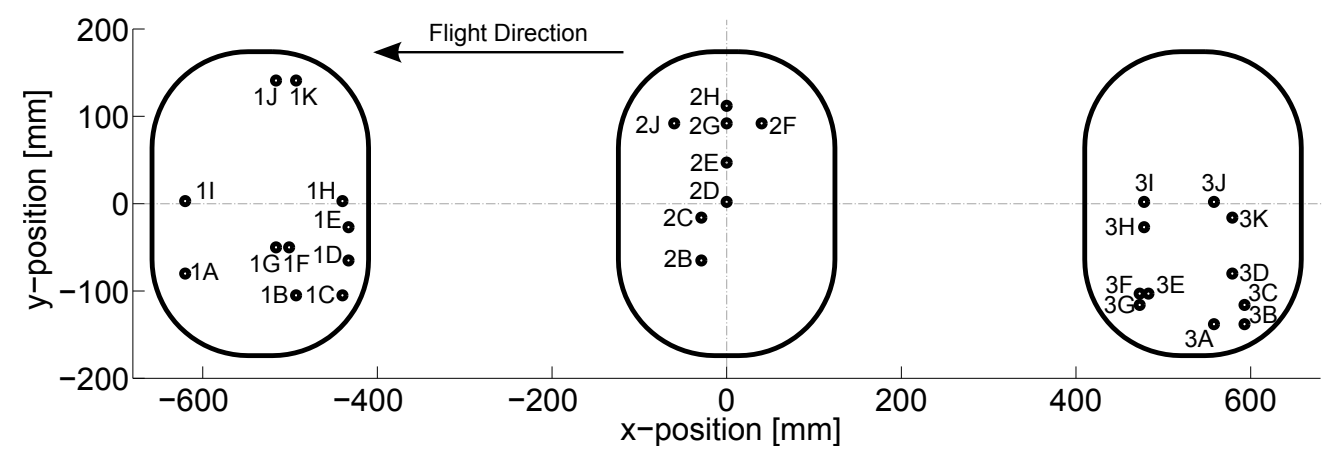

Figure 3. Kulite installation details (array-fixed coordinates)

In figures 4 and 5 one can see the installation areas of the second sensor type, flush mounted Brüel \& Kjaer aircraft surface microphones (Type 4948-W-003). Figure 4 presents the aft measurement region, again coloured in green. In this area a number of 68 microphones were installed. Moreover, the front measurement region is shown in figure 5. The flush mounted microphones were installed in groups of two, three or five sensors as well as stand-alone microphones. A group of three sensors is always installed in the form of a so called Triplet and all other arrangements are composed of stand-alone microphones. These two basic types are drafted in figure 6, where the microphones are depicted as black dots. One can see that the microphones are surrounded by a gray area. This denotes the backing material of the microphones, a rubber like mat of $d \approx 4 \mathrm{~mm}$ thickness which can be glued on the fuselage, triangular in shape for the triplets and circular for the stand-alone microphones. 


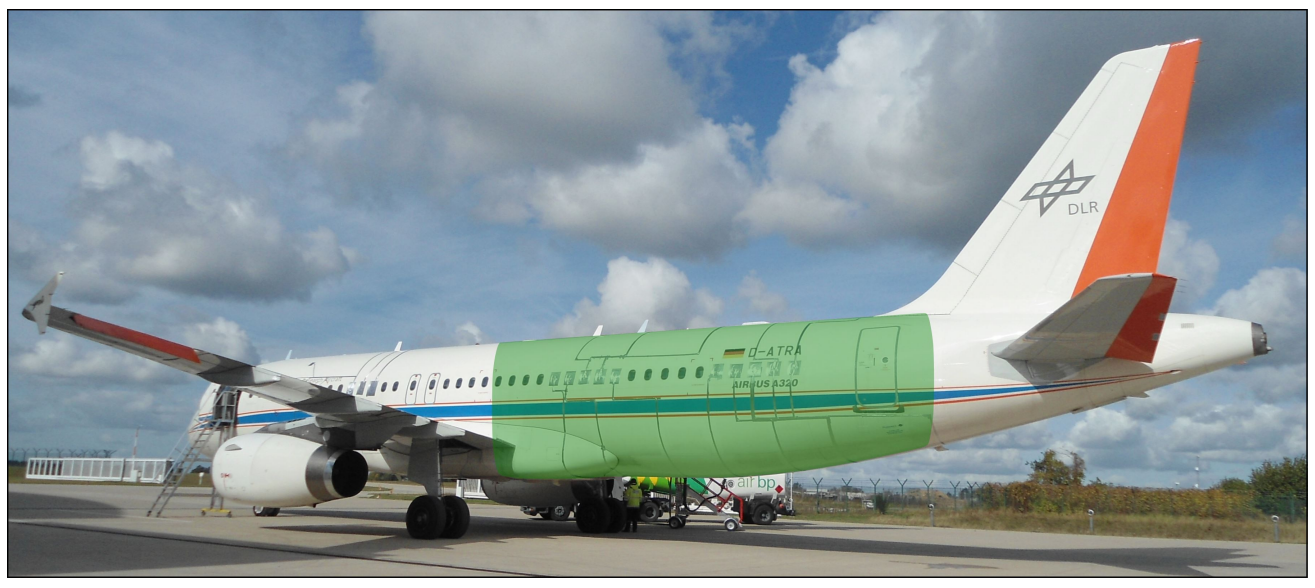

Figure 4. Microphone measurement region, aft

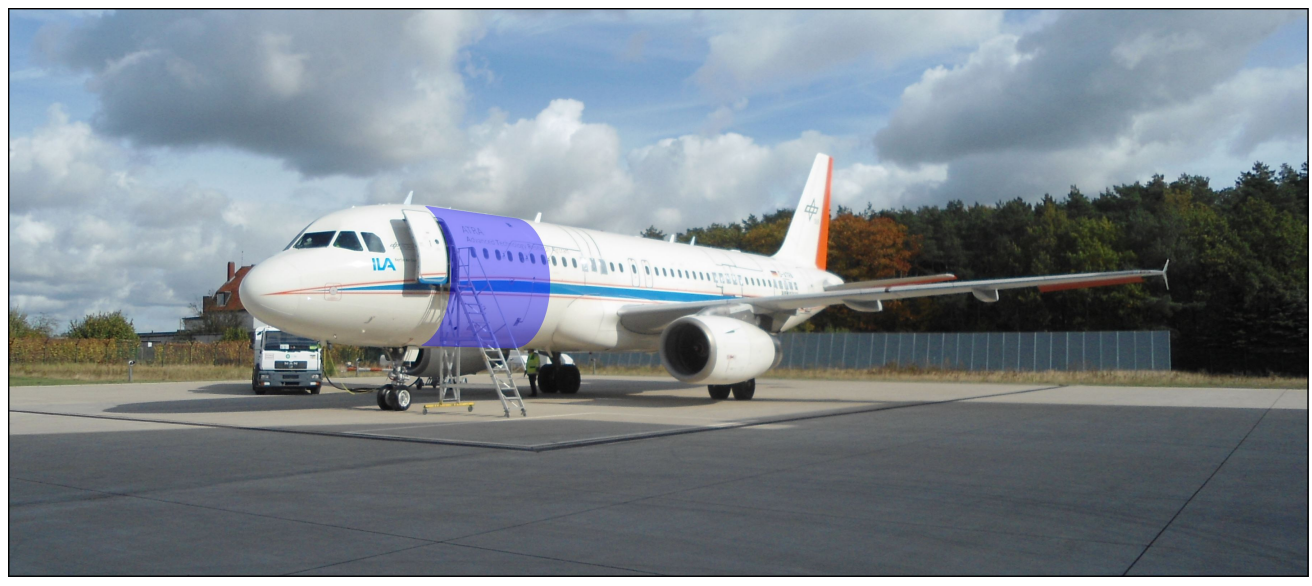

Figure 5. Microphone measurement region, front
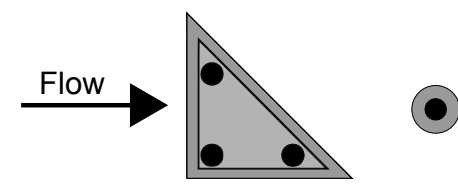

Figure 6. Sketch of flush mounted microphones (black dots) with backing material (gray) 


\section{Flight test data analysis}

In the flight test data analysis section, firstly, a comparison between all different sensors and installation types, located next to each other is given in terms of auto-spectra. This analysis is used for the definition of a frequency confidence range, where all data are comparable. Secondly, the influence of different positions on the fuselage is examined at steady cruise flight condition, which is Ma $=0.78$ at FL 350. Thirdly, a comparison of the data, measured at different flight configurations is presented, focused on the effect of Mach number and altitude variation. Therefore, some test flight configurations are picked out of the whole test flight matrix, which is presented in figure 7. Configurations of interest are highlighted with a gray ellipse and they are in the range of $\mathrm{Ma}=0.72 \ldots 0.82$ and FL 310...FL 390. Data for each fuselage region (front, middle, aft) is available as denoted by the coloured symbols. Furthermore, in figure 8(a) one can see the global coordinate system for the definition of the sensor positions in x-direction and in fig. 8(b) the definition of the azimuthal position. For TBL parameter calculation the origin of the a/c nose at $\mathrm{x}=2.5 \mathrm{~m}$ is taken into account.

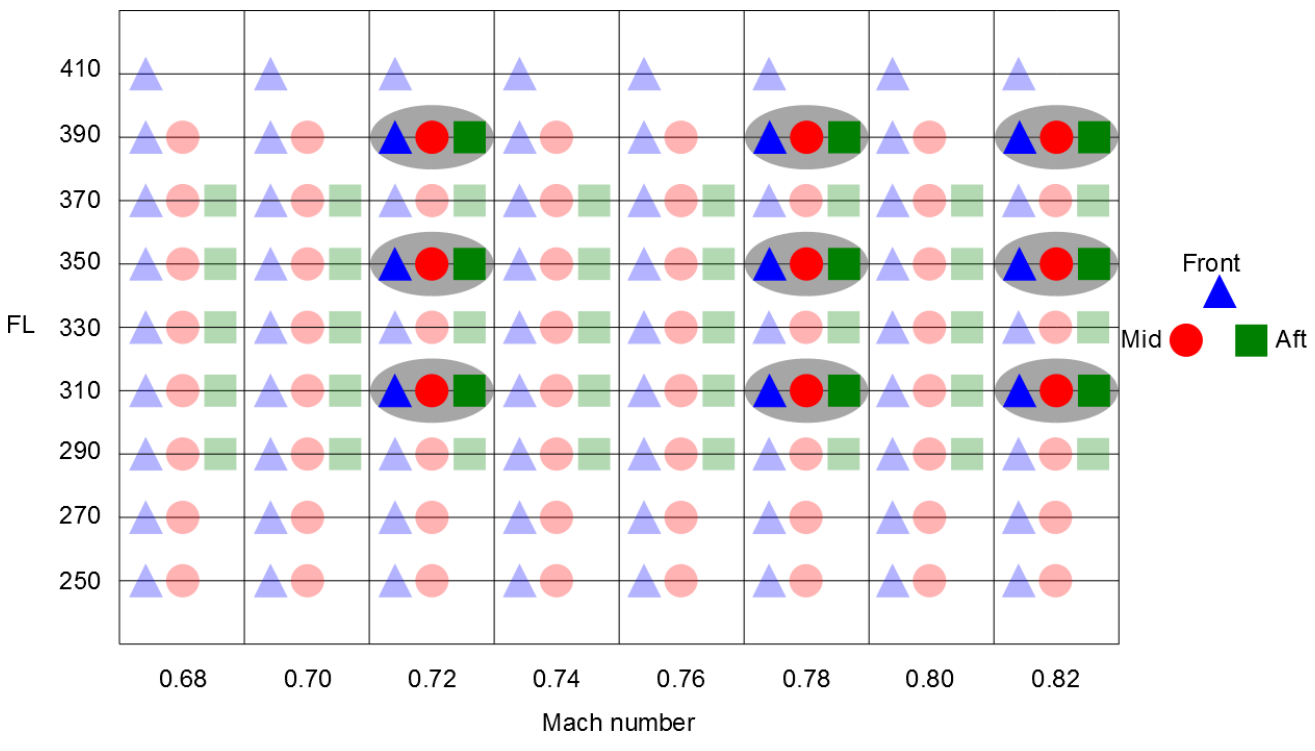

Figure 7. Test flight configurations and data availability

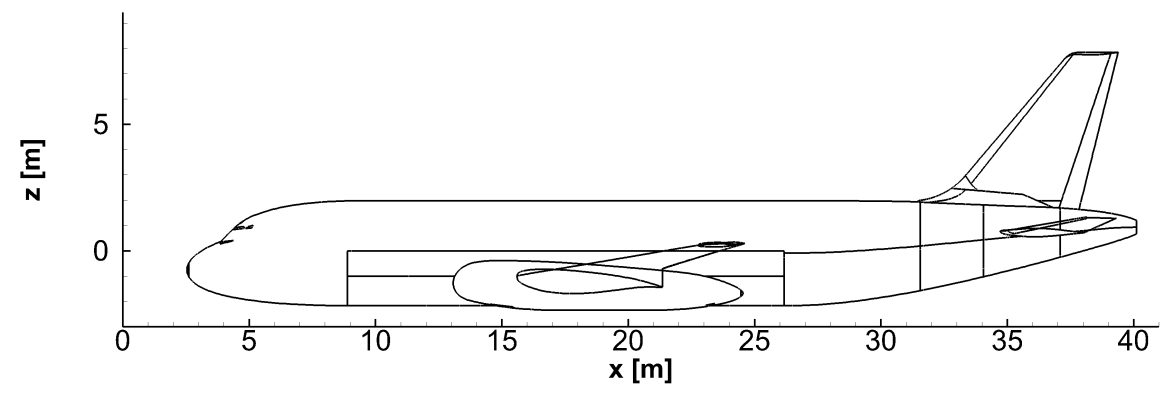

(a) Global coordinate system (x/z-direction)

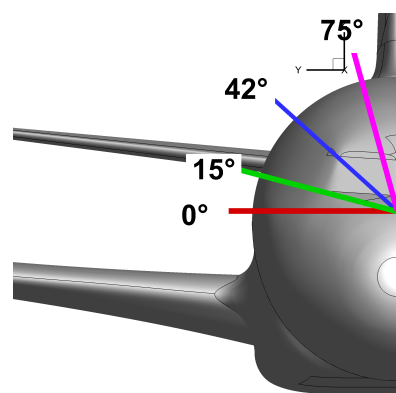

(b) Azimuthal coordinates

Figure 8. Global coordinate system

\section{A. Frequency confidence range}

One of the first steps in the analysis is, to check the similarity of the results given by different sensors, to find a frequency confidence range. In the green areas in figures 2 and 4, directly behind the wing and near the center 
line of the aircraft all sensors and installation types are contiguous (Kulites in Kulite windows, microphones as Triplets and flush mounted stand-alone microphones). A comparison of the measured auto-spectra from each sensor is given in figure 9(a). The microphone sensor data (green and red) are CoRCos corrected, due to the sensor surface area. The plotted line is the final result, depicted with an error bar caused by uncertainties in the correction method. The lower end of the error bar is the uncorrected signal and the upper end is the standard Concos corrected signal, which is in level too high above $f>4000 \mathrm{~Hz}$ due to unknown reasons. The final result is the energetic mean value between these borders and seems to give best results because it satisfies the expected $\omega^{-1}$ slope. Analysing the data, one can see good agreement for the measured spectral densities between $f=400 \ldots 3000 \mathrm{~Hz}$. Between $1.5 \mathrm{kHz}$ and $3.5 \mathrm{kHz}$ the Kulite measurement results (blue line) deviate by about $2 \mathrm{~dB}$ from the microphones (red and green lines). Above and below this frequency range of good agreement, large differences are evident. In the range between $f=100 \ldots 400 \mathrm{~Hz}$, the autospectrum level of the stand-alone microphone increases with decreasing frequency in comparison to the Kulite and Triplet microphone data. The main reason for this is the installation situation and the distance to the front edge of the backing material (cf. fig. 6). This front edge shapes a turbulence inducing step directly upstream the stand-alone microphone and the two upstream microphones of the triplet group. Only the downstream microphone is not affected by this little step and therefore equal in level with the Kulite data. In the high frequency range above $f>3000 \mathrm{~Hz}$, again a large deviation between the sensors can be observed, but in this case the microphones are nearly equal in level and the Kulite sensor data describe a much steeper roll-off than the microphone data. This little deviation in the microphone data is due to the afore mentioned step of the backing material and as consequence of the low frequency increase of the stand-alone microphone auto-spectrum. Furthermore, the steep roll-off of the Kulite auto-spectrum is as well due to the installation situation and likely caused by a Helmholtz resonance problem.

All beforehand mentioned observations are distinctive for all "three-symbol-configurations" listed in figure 7. Furthermore, data analysis showed that the engine setting has no influence on the described basic sensor characteristics. Finally, the whole frequency range $f=100 \ldots 10000 \mathrm{~Hz}$ is covered by the measurement data. Kulite data confidence range is between $f=100 \ldots 3000 \mathrm{~Hz}$, stand-alone and upstream microphone data is trustworthy in the range of $f=400 \ldots 10000 \mathrm{~Hz}$ and data from the most downstream located Triplet microphone is supposed to be reliable in the whole presented frequency range. This knowledge was used to establish a combined reference spectrum, which is presented in figure 9(b) for the aft region. An error bar is also applied in the low frequency region due to the deviation between B\&K microphone and Kulite data $(\max . \approx 2 \mathrm{~dB})$.

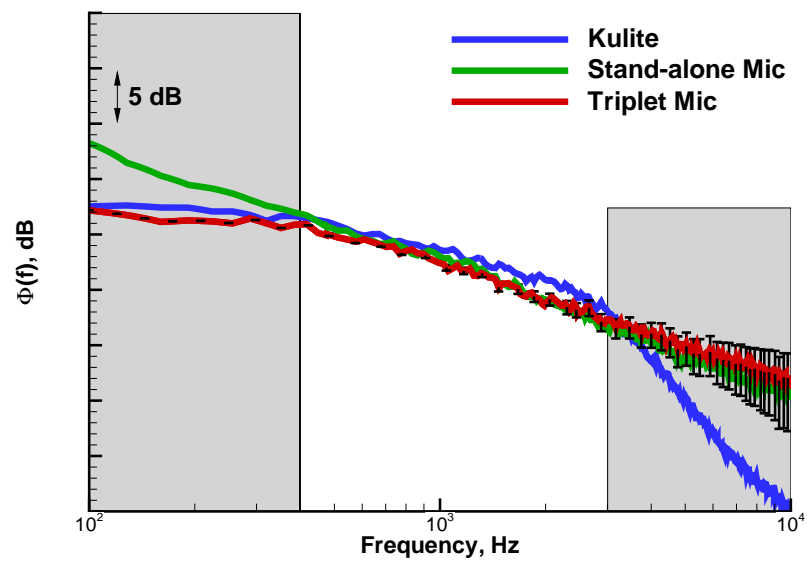

(a) Sensor comparison

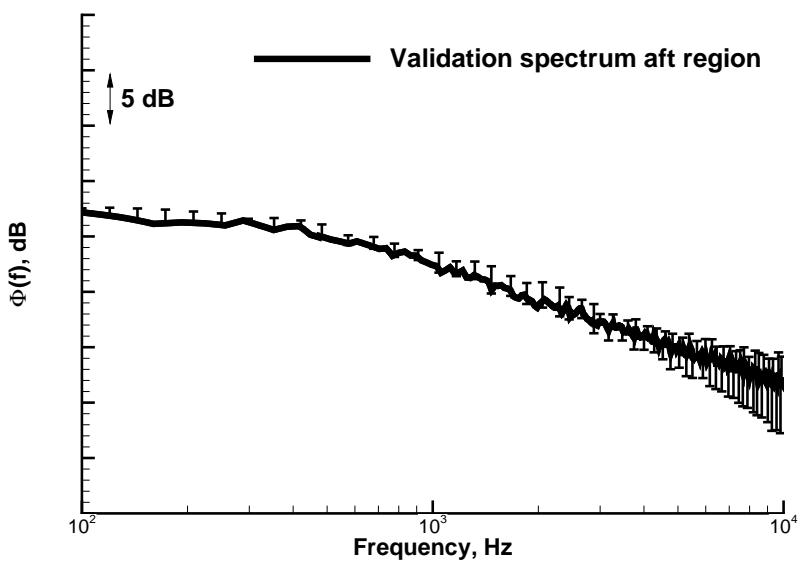

(b) Validation spectrum aft region

Figure 9. Sensor comparison and validation spectrum; Ma 0.78, FL $350, x \approx 26 \mathrm{~m}$, engine idle

\section{B. Effect of Position variation}

The sensors in the front area are installed at $\mathrm{x}=9.2 \mathrm{~m}$, the window in the middle is at $\mathrm{x}=15.0 \mathrm{~m}$ and the most backwards Kulites are located at $\mathrm{x}=26.0 \mathrm{~m}$, compare fig. 8(a) for definition of $\mathrm{x}$. In figure 10(a) data from FL 350 and Ma 0.78 are presented. It is $\mathrm{x}=9.2 \mathrm{~m}$ blue, $\mathrm{x}=15.0 \mathrm{~m}$ red and $\mathrm{x}=26.0 \mathrm{~m}$ green lines 
respectively. The trend is as expected, with increasing x-value, the spectra increase in the low frequency region and decrease in the higher frequency region. This reflects the development of the TBL thickness, the larger $\mathrm{x}$, the thicker the TBL and therefore, bigger structures dominate the spectra. An equivalent trend can also be seen in the B\&K microphone data, which are plotted in figure $10(\mathrm{~b})$ for the front $(x \approx 9.9 \mathrm{~m})$ and aft $(x \approx 26.5 \mathrm{~m})$ positions. One can see data from stand-alone microphones, plotted in solid lines and the green dashed line denote data from a downstream Triplet microphone. The Triplet microphone shows trustworthy data in the frequency range between $f=100 \ldots 10000 \mathrm{~Hz}$. All other microphone data are affected by the step upstream the sensor between $f=100 \ldots 400 \mathrm{~Hz}$. However, the effect of increasing $\mathrm{x}$ is equal for all beforehand mentioned sensor types.

Beside this comparison, further comparison can be made with microphones located between the trailing edge of the wing and the horizontal tail plane (HTP) / vertical tail plane (VTP) zone. This region is shown in figure 4 and the chosen sensors for analysis are located in a straight line above and parallel to the passenger windows at an azimuthal angle of $42^{\circ} \ldots 51^{\circ}$ (cf. fig. 8(b)). In figure 11, the measured auto-spectra are compared, beginning at $\mathrm{x}=22.1 \mathrm{~m}$ and ending at $\mathrm{x}=30.5 \mathrm{~m}$. Microphones are distributed equidistantly. At first glance it is striking that there is nearly no change in the spectra with varying $\mathrm{x}$. However, this is an expected result, which can be partly explained with the development of the local boundary layer thickness $\delta_{l}$. From $\mathrm{x}=22.1 \ldots 30.5 \mathrm{~m}$ the thickness of the boundary layer is increasing by roughly $25 \%$ (flat plate estimate), corresponding to a level increase of order $1 \mathrm{~dB}$ which cannot be resolved by the respective measurements (lies within the data scattering). In contrast, the local boundary layer thickness is always doubled from front to mid and from mid to aft as well, therefore the increase of $3 \mathrm{~dB}$ in level at low frequencies can be explained, compare fig. 10(a).

In summary, it can be stated that there is a distinct change in the spectra with varying $\mathrm{x}$-value from the nose to the wing's trailing edge (Kulite comparison), but in the area behind the wing up to the HTP/VTP zone, no change in spectral shape and level can be seen. Reasons for that were given beforehand on the basis of the development of local boundary layer thickness.

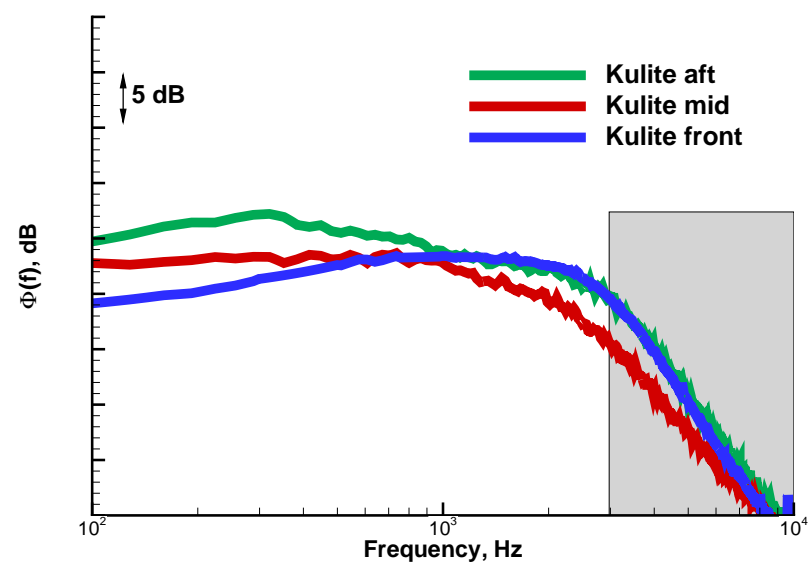

(a) Kulite, $x$ variation, whole fuselage

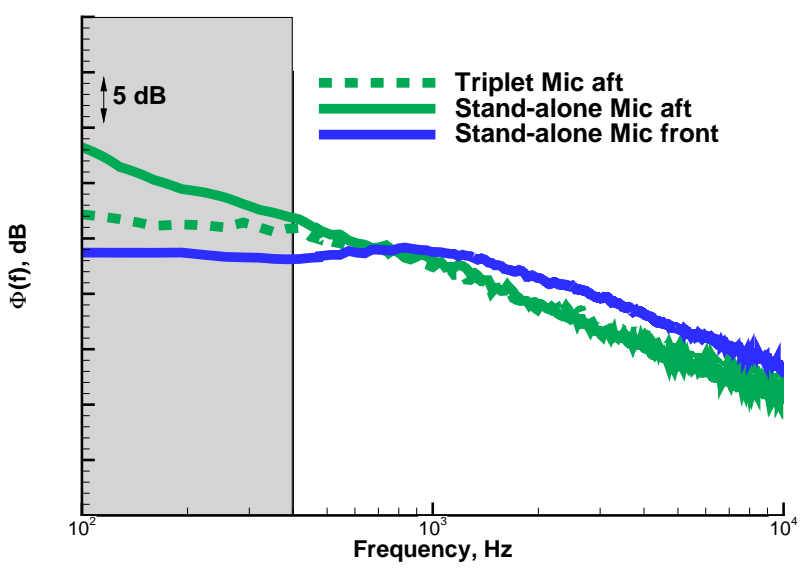

(b) Microphones, $\mathrm{x}$ variation, whole fuselage

Figure 10. Position variation on the fuselage, Kulites and B \& $\mathrm{K}$ microphones

\section{Effect of Mach number- and flight level variation}

Figures 12(a) and 12(b) show the influence of Mach number and flight level variation on the auto-spectra $(\mathrm{x} \approx 26.0 \mathrm{~m})$. Again, all available sensor types are implied in this analysis. One can see the effect of a varying flight level in figure $12(\mathrm{a})$ at constant Mach number $(\mathrm{Ma}=0.78)$, the red dashed lines depict data recorded at FL 350 (standard cruise flight) and the solid black lines are FL 310. Lowering the flight altitude by $4000 \mathrm{ft}$ leads to a broadband noise increase by about $2 \mathrm{~dB}$, which can be explained by a higher air density at the lower flight level. These findings correspond to the observed results for measured cabin noise at the same test conditions. ${ }^{6}$ Beside this, a similar $2 \mathrm{~dB}$ noise increase can be seen in figure 12(b), where the Mach number is varied from $\mathrm{Ma}=0.72$ to $\mathrm{Ma}=0.78$ at a constant flight level of FL 310 . 


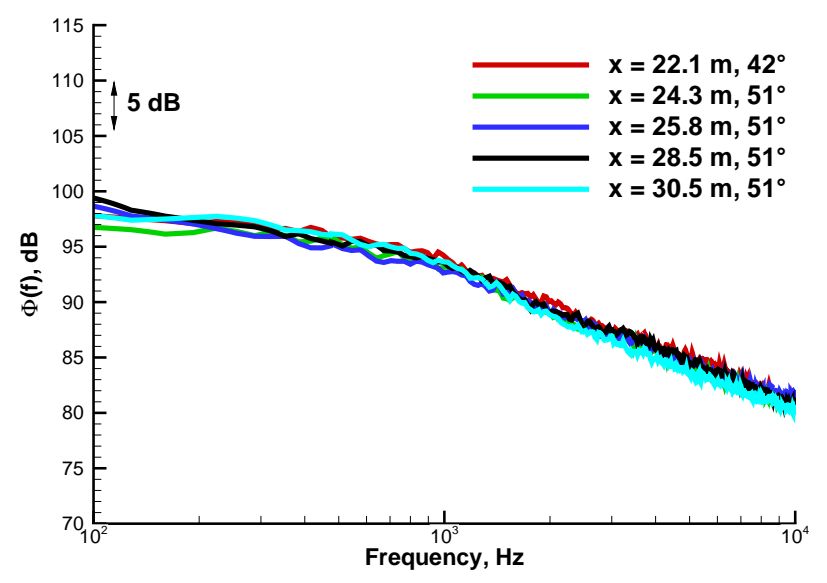

Figure 11. Position variation on the fuselage, microphones aft region

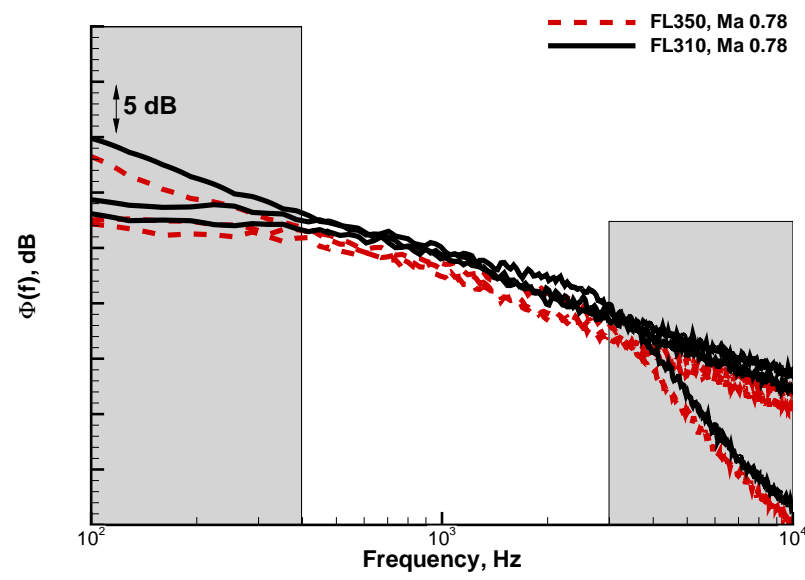

(a) Sensors, variation in flight level (FL350 vs. FL310)

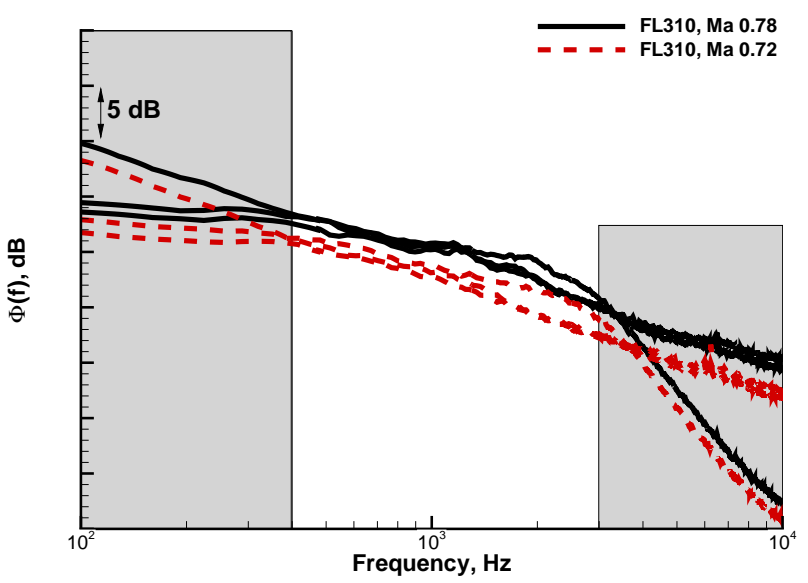

(b) Sensors, variation in Mach number (Ma0.78 vs. Ma0.72)

Figure 12. Variation of flight level and Mach number for different sensors 


\section{CFD analysis and TBL parameter extraction}

Detailed TBL characteristics were not assessed during the flight tests. Therefore, a CFD analysis of the DLR ATRA flow field has been conducted to supplement the analysis. The CFD calculations include a full aircraft configuration considering wings with belly fairing and flap track fairings, winglets, VTP and HTP. Only the engines with pylon were neglected. The DLR CFD code TAU was used applying the SpalartAllmaras Original turbulence model $(\mathrm{SAO})^{22}$ as well as Reynolds Stress model (RSM). ${ }^{22}$ In table 1 all simulation cases are listed.

Table 1. CFD flight conditions

\begin{tabular}{|c|c|c|c|c|c|}
\hline Case & FL $[100 \mathrm{ft}]$ & Ma $[-]$ & $\alpha\left[^{\circ}\right]$ & TAS $[\mathrm{m} / \mathrm{s}]$ & $\Delta$ ISA \\
\hline 1 & 350 & 0.82 & $2^{\circ}, 3^{\circ}, 4^{\circ}$ & 247 & $0 \mathrm{~K}$ \\
2 & 310 & 0.82 & $0.9^{\circ}$ & 245 & $0 \mathrm{~K}$ \\
3 & 310 & 0.78 & $1^{\circ}$ & 234 & $0 \mathrm{~K}$ \\
4 & 350 & 0.78 & $2^{\circ}$ & 237 & $0 \mathrm{~K}$ \\
\hline
\end{tabular}

Data for Case 4 is shown in the following, because this is standard cruise flight condition. Details about the pressure coefficient $\left(c_{p}\right)$, the local boundary layer thickness $\left(\delta_{l}\right)$, the local flow direction and the turbulence kinetic energy $(k)$ were analysed and are partly presented in the following, with a focus on the last two properties.

\section{A. Analysis of pressure coefficient $\left(c_{p}\right)$}

An analysis of the pressure coefficient around the whole A320 fuselage wsa conducted, to identify and quantify regions with and without pressure gradients. This analysis was done in detail at all sensor locations. Regions with strong pressure gradients, especially in the cockpit-, wing- and HTP/VTP area were identified as well as region without noteworthy flow alteration. Sensors were located in all these areas. The analysis results gave a first hint that the flow around the fuselage is very complex with acceleration and deceleration areas that influence the measured auto-spectra in level and shape.

\section{B. Analysis of local boundary layer thickness $\left(\delta_{l}\right)$}

Furthermore, parameters like $\delta_{l}, U_{\tau}$ and $U_{e}$ were analysed. Two methods were applied to estimate the local boundary layer thickness. On the one hand, the velocity profiles were analysed and the local velocity at the boundary layer edge based on the surface pressure coefficient $c_{p}$ was determined to achieve local boundary layer thickness at $99 \% U_{e} \cdot{ }^{23}$ On the other hand, a method which is focused on the total pressure $\left(p_{t o}\right)$ was applied. By approaching the fuselage surface from the far field, perpendicular to the surface, a loss in total pressure is present near the surface. The local boundary layer thickness was defined at $p=0.99 \cdot p_{t o}$. Both methods show good agreement over large fuselage areas.

CFD calculated $\delta_{l}$ were compared to theoretical estimates based on flat plate estimates according to SCHLICHT$\mathrm{ING}^{24}$ because some of the original auto-spectra models are fed by that theoretical data, i. e.

$$
\delta=0.37 \frac{x}{R e^{1 / 5}} \text { with } R e=\frac{x \cdot U_{\infty}}{\nu} .
$$

Deviation of $\delta_{l}$ are between $35 \%$ underestimation up to $66 \%$ overestimation by the flat plate method compared to CFD.

\section{Analysis of local flow direction}

In this section the focus is on the CFD calculated local flow direction and a quick comparison to previously published measurement data is discussed. The local convection direction of the flow was analysed by HAXTER $^{3}$ at $\mathrm{x}=15 \mathrm{~m}$, using detailed Kulite cross-correlation data. HAXTER found out that the convection direction is $\vartheta_{F T} \approx 10^{\circ} \ldots 18^{\circ}$ between $f \approx 800 \ldots 5000 \mathrm{~Hz}$, relative to the aircraft x-axis. In the frequency range of $f \approx 800 \ldots 2100 \mathrm{~Hz}$, the convection direction is $\vartheta_{F T} \approx 10^{\circ} \ldots 13^{\circ}$. At higher frequencies, $f>2100 \mathrm{~Hz}$, 
the convection direction is measured as $\vartheta_{F T} \approx 14^{\circ} \ldots 18^{\circ}$.

Comparable values can also be found in the CFD calculation results. In figure 13(a) values of the flow angle, along slices parallel to the x-axis for different azimuthal angles, are plotted (cf. fig. 8(b)). The presented flow angle is analysed at a distance of $\mathrm{d}=0.1 \mathrm{~m}$ above the fuselage. In the area $\mathrm{x} \approx 9 \ldots 30 \mathrm{~m}$, the modulus of the flow angles increases with decreasing azimuthal angle of the slices, thus with decreasing distance to the wing. This is according to expectation because the influence of the wing and thus the displacement of the fluid diminishes with increasing distance (azimuthal angle). At maximum presented azimuthal angle the influence of the wing at $\mathrm{x}=9 \ldots 18 \mathrm{~m}$ is nearly negligible and the flow direction equals the angle of attack $\left(\alpha=2^{\circ}\right)$. Furthermore, in the front part of the fuselage $(\mathrm{x}=2.5 \ldots 5 \mathrm{~m})$ strong positive flow angles are present, which is an effect of the cockpit shape.

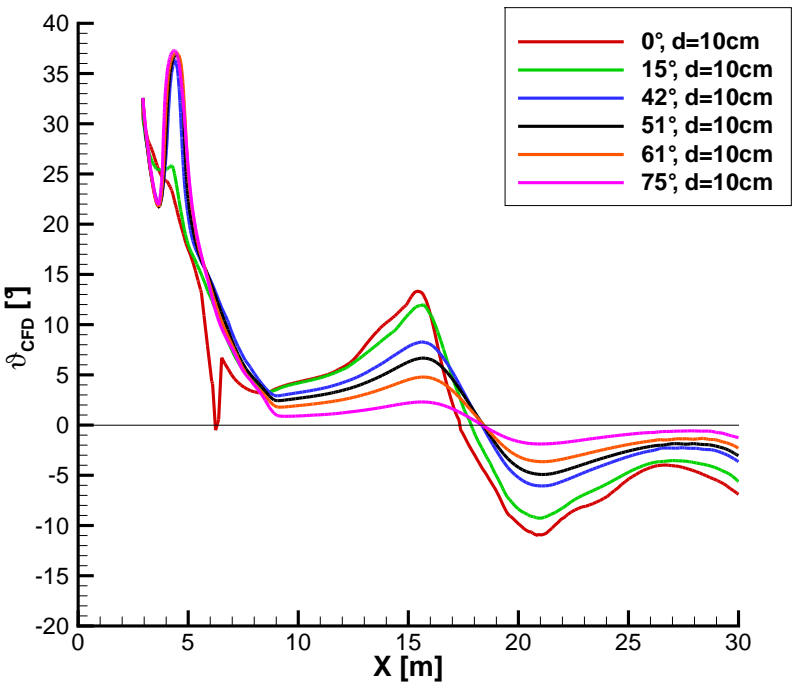

(a) Flow angle $\vartheta_{C F D}$ over fuselage, $\mathrm{d}=0.1 \mathrm{~m}$

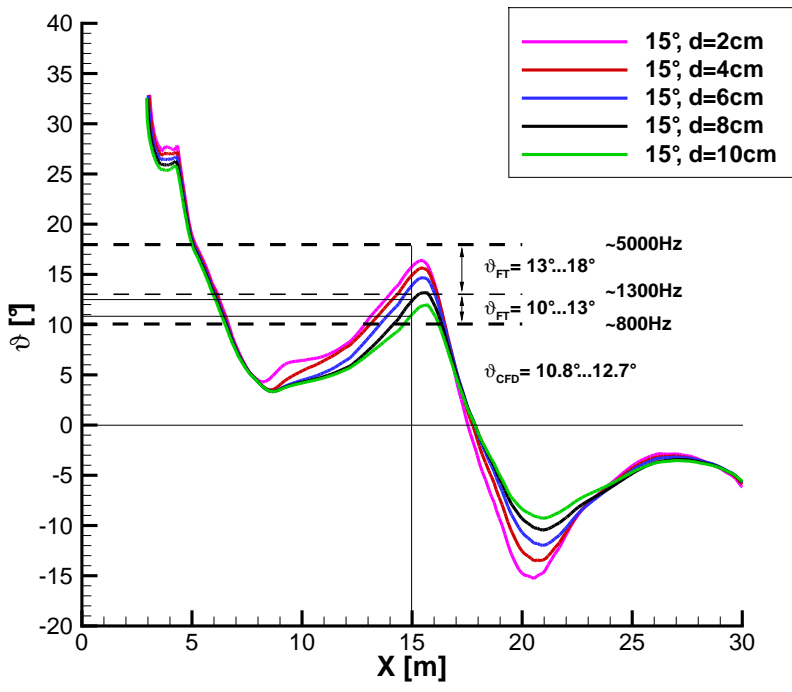

(b) Flow angle $\vartheta_{C F D}$, window plane $(\mathrm{d}=0.02 \ldots 0.1 \mathrm{~m})$, compared with convection direction from correlation analysis $\left(\vartheta_{F T}\right)$, HAXTER $^{3}$

Figure 13. Flow angle $\vartheta$ over fuselage

For a comparison with the measurement results, the $15^{\circ}$ azimuthal angle plane is analysed more detailed in figure 13(b). Extracted flow angles are shown for different distances $\mathrm{d}=0.02 \ldots 0.1 \mathrm{~m}$ in steps of $0.02 \mathrm{~m}$ perpendicular to the fuselage surface. At the center of the Kulite array $(\mathrm{x}=15 \mathrm{~m})$, the flow angle $\vartheta_{C F D}$ increases with decreasing fuselage distance. A minimum value is reached at $\mathrm{d}=0.1 \mathrm{~m}$ with $\vartheta_{C F D} \approx 10.8^{\circ}$ and the maximum is located at $\mathrm{d}=0.02 \mathrm{~m}$ with $\vartheta_{C F D} \approx 17^{\circ}$. This development is according to expectation because the flow direction is twisted with increasing wall distance until it reaches the flow direction of the free stream.

In the previous analysis, the local boundary layer thickness was found to be $\delta_{l} \approx 0.13 \mathrm{~m}$ at $\mathrm{x}=15 \mathrm{~m}$. The flight test data correlation analysis showed that the convection velocity angle is $\vartheta_{F T} \approx 10^{\circ} \ldots 13^{\circ}$ in the frequency range of $f \approx 800 \ldots 2100 \mathrm{~Hz}$. Due to the fact of having most of the energy located in that frequency range, one can conclude that the CFD calculated flow direction at $\mathrm{d}=0.08 \ldots 0.10 \mathrm{~m}$ can be assumed to represent that frequency range in an appropriate manner. For distance $\mathrm{d}=0.08 \ldots 0.1 \mathrm{~m}$, the flow angle is $\vartheta_{C F D} \approx 10.8^{\circ} \ldots 12.7^{\circ}$, respectively and this correlates with the measured convection angle $\vartheta_{F T} \approx 10^{\circ} \ldots 13^{\circ}$ for $f \approx 800 \ldots 2100 \mathrm{~Hz}$ in the flight test data.

\section{Analysis of turbulence kinetic energy (TKE)}

Finally, some results of the calculated turbulence kinetic energy $(k)$ are presented in this section. $k$ is calculated as stated in Eq. (19) and constitutes the kinetic energy per unit mass of the turbulent fluctuations $u_{i}^{\prime}$ in a turbulent flow

$$
k=0.5\left(\overline{\left(u_{x}^{\prime}\right)^{2}}+\overline{\left(u_{y}^{\prime}\right)^{2}}+\overline{\left(u_{u}^{\prime}\right)^{2}}\right) .
$$


The analysis of $k$ was performed for a large area behind the wing trailing edge and above it. In figure 14 this area is presented with the respective flight test measurement positions on the fuselage. This area is subdivided into two smaller zones, firstly with Triplets $1 \ldots 3$ (TL1, TL2, TL3) and secondly with Triplets 5, 8 and 11 (TL5, TL8, TL11). Each Triplet is a group of three microphones, as introduced in chapter III. Here, the variation of azimuthal angle and the variation of running length is of interest. Results are always presented together with the local boundary layer thickness $\left(\delta_{l}\right)$ and pressure distribution $\left(c_{p}\right)$. Results of zone one are given in figures $15(\mathrm{a})-15(\mathrm{~d})$ and results of zone two are presented in figures $15(\mathrm{e})-15(\mathrm{f})$. In these figures one can see the cut through the fuselage as a horizontal black line and the magenta line above denotes the local boundary layer thickness. The arrows with the respective Triplet numbers designate their position on the fuselage. Furthermore, the $k$ and $c_{p}$ distribution is shown by a coloured background.

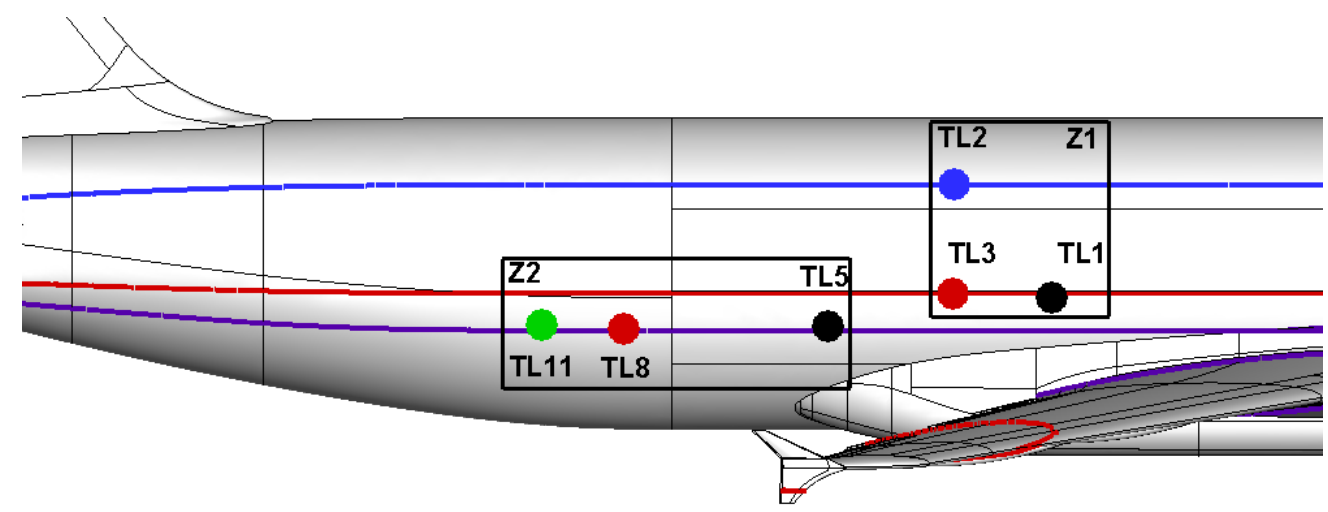

Figure 14. Fuselage positions for analysis of $k$

According to plate theoretical estimates and semi-empirical models, TL1, TL2 and TL3 should show very similar results in terms of auto-spectra, due to similar running length and $\delta_{l}$. Taking the distribution of the pressure coefficient into account, one can see in fig. 15(b) and fig. 15(d) a slightly decelerated flow and a slightly thickened boundary layer from TL1 to TL2/TL3, which should have no significant influence on the auto-spectra. Going one step further and analysing $k$ (fig. 15(a) and 15(c)), considerable differences are obvious between TL1, TL2 and TL3. TL1 and TL3 are located in the wing wake and especially TL1 is located near a maximum of $k$. Whereat TL2 is positioned in an area with very low $k$ values, due to its position further up on the fuselage, far away from the wing.

Beside this, the zone two Triplets (TL5, TL8, TL11) are also located in an area without strong pressure gradients, as can be seen in figure 15(f). The flow at TL5 is somewhat decelerated and in contrast, the flow at TL8 and TL11 is a bit accelerated. However, the local thickness of the boundary layer is almost constant. Taking again the $k$ distribution in fig. 15(e) into account, one can see an increase of energy near TL5 and a decreasing amount of turbulence kinetic energy with increasing running length. Therefore, TL8 and TL11 are located in an area of less TKE than TL5 is.

These determinations are important for the further analysis of measured auto-spectra and calculated semiempirical models in the subsequent chapter. 


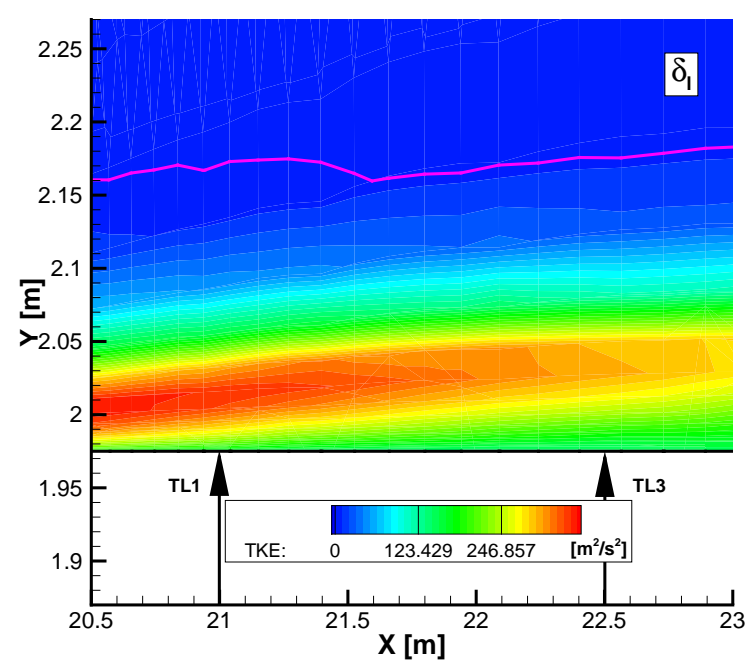

(a) TKE distribution at TL1, TL3

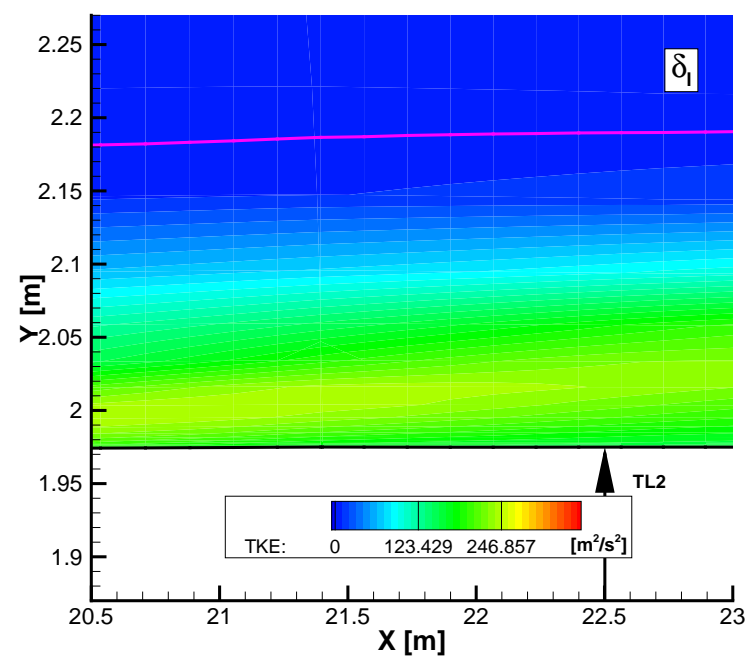

(c) TKE distribution at TL2

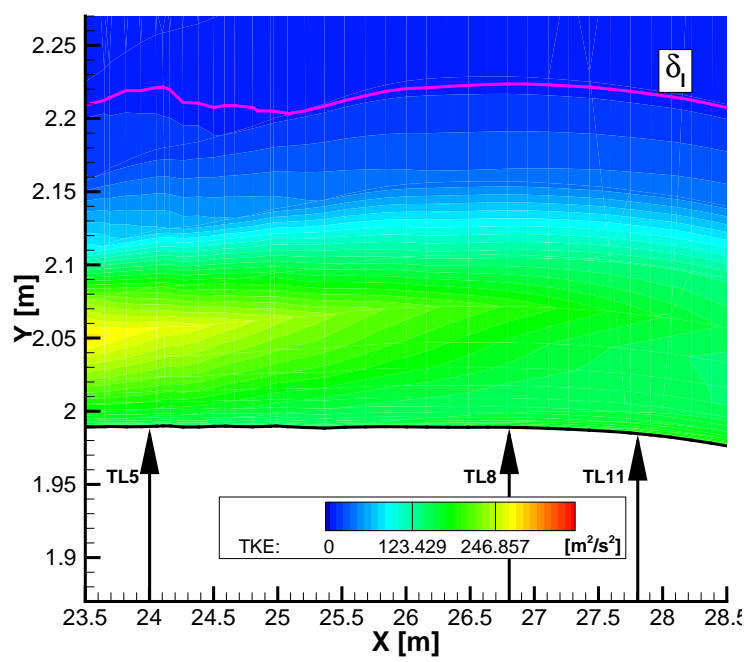

(e) TKE distribution at TL5, TL8 and TL11

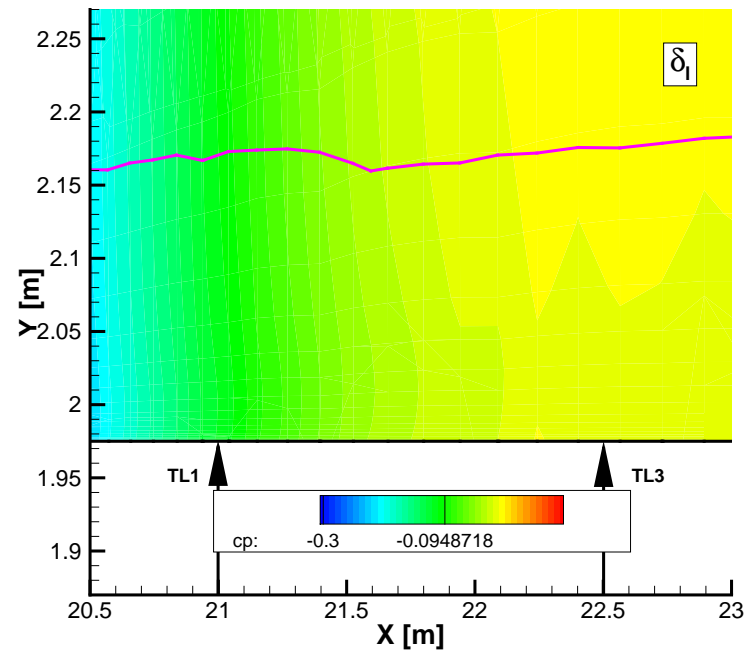

(b) $c_{p}$ distribution at TL1, TL3

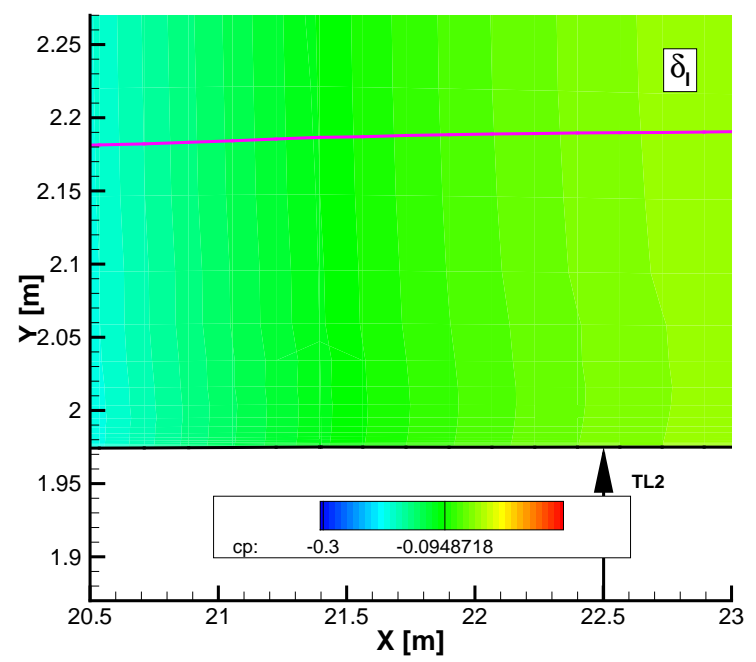

(d) $c_{p}$ distribution at TL2

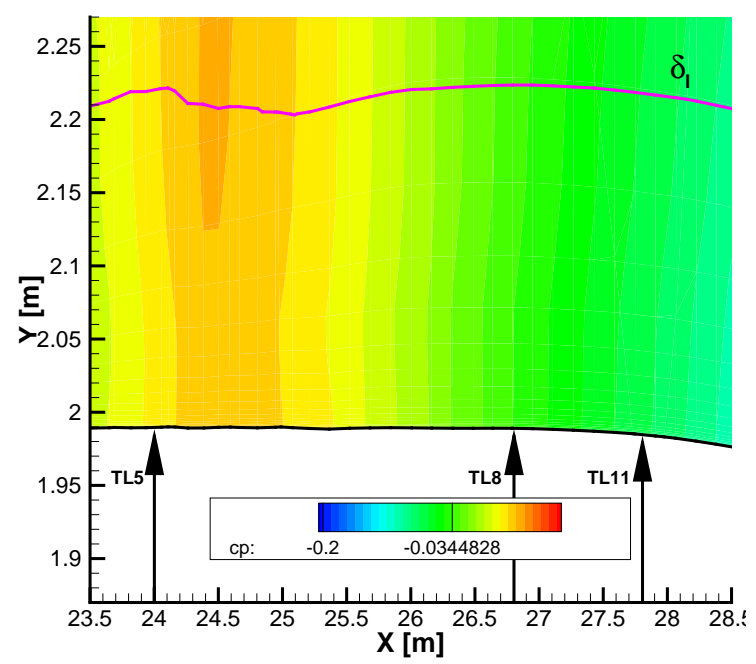

(f) $c_{p}$ distribution at TL5, TL8 and T111

Figure 15. TKE, $c_{p}$ and $\delta_{l}$ comparison at TL1, TL2 and TL3 


\section{Comparison of flight test data and semi-empirical models}

In this section an overview about the auto-spectra, measured during the flight test campaigns is given in comparison to the calculated auto-spectra, based on semi-empirical models. Data from different sensors (Kulite pressure transducers, flush mounted microphones), at different locations are presented and compared with the semi-empirical model results. All data are taken from configurations at FL 350 and Ma $=0.78$. The first step in the following sections is, to calculate the semi-empirical auto-spectra models based on TBL parameters derived on flat plate estimates. Afterwards, this comparison is extended and the auto-spectra models are calculated based on CFD data, which was analysed in the previous chapter.

\section{A. Models based on flat plate estimates}

In figure 16(a), a comparison between the measured Kulite results in the front section (figure 2, blue area) and five semi-empirical models is shown. The presented configuration is standard cruise flight condition at FL 350 and $\mathrm{Ma}=0.78$. A closer view on the plotted Kulite results shows a scatter of approx. $2 \mathrm{~dB}$ below $f<2000 \mathrm{~Hz}$ and above $f>6000 \mathrm{~Hz}$ in the data, in between it is less than $2 \mathrm{~dB}$.

Furthermore, a comparison of the before mentioned set of data is shown in figure 16(b) with the four remaining semi-empirical models. Comparing figure 16(a) and 16(b) one can see that the Goody model fits the absolute values of the Kulite data best in a frequency range up to $f<2000 \mathrm{~Hz}$. The steep roll-off, which can be seen in the Kulite data above $f>2000 \mathrm{~Hz}$ cannot be approximated by any presented semi-empirical model. However, this seems to be an artefact of the Kulite installation situation, triggered by a Helmholtz resonance phenomenon and is therefore not within the confidence range of the Kulite data.

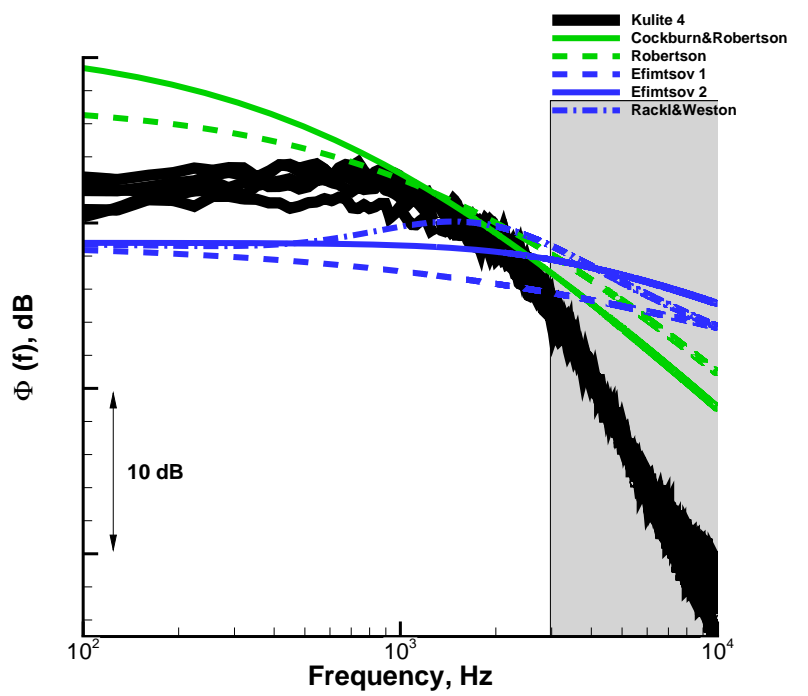

(a) Kulites FL350, $\mathrm{Ma}=0.78, \mathrm{x}=15 \mathrm{~m}$

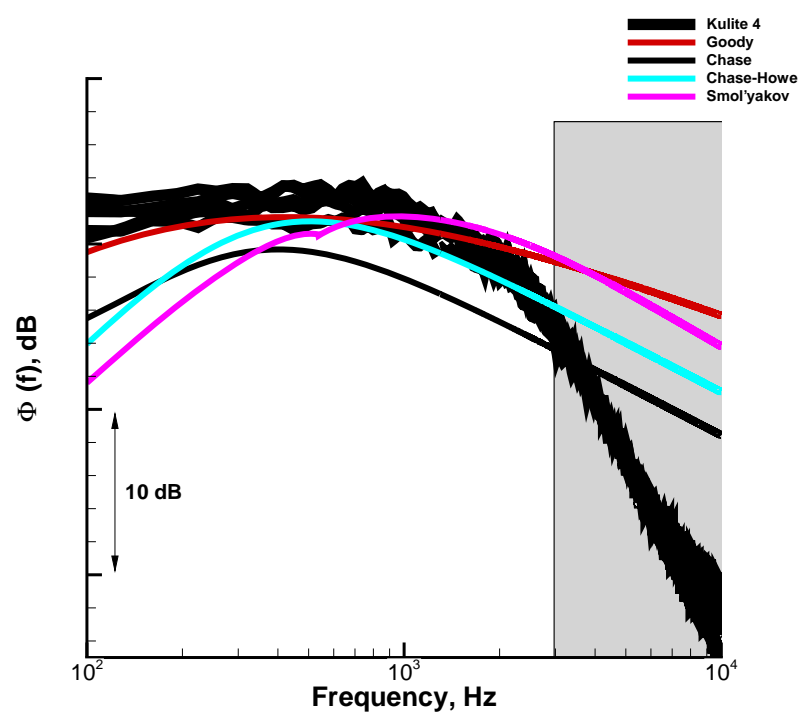

(b) Kulites FL350, $\mathrm{Ma}=0.78, \mathrm{x}=15 \mathrm{~m}$

Figure 16. Kulite data comparison with semi-empirical models

In figure 17, measurement data of the positions, specified in figure 14 are shown in comparison with the semi-empirical Goody auto-spectra, which is supposed to fit the data best. One can see in fig. 17(a) and fig. 17(b), which is zone one and zone two respectively, that the estimates made with the Goody model does not fit the measured data. Neither in level nor in shape. The model predicts the same spectrum for all positions, as previously mentioned. The attempt to improve these results by using flow parameters from a CFD calculation is shown in the following.

\section{B. Models based on CFD}

In the former section, semi-empirical auto-spectra models, based on flat plate estimates, were presented in comparison with measured data. In figures 18(a) and 18(b) the semi-empirical model of Goody (based on 


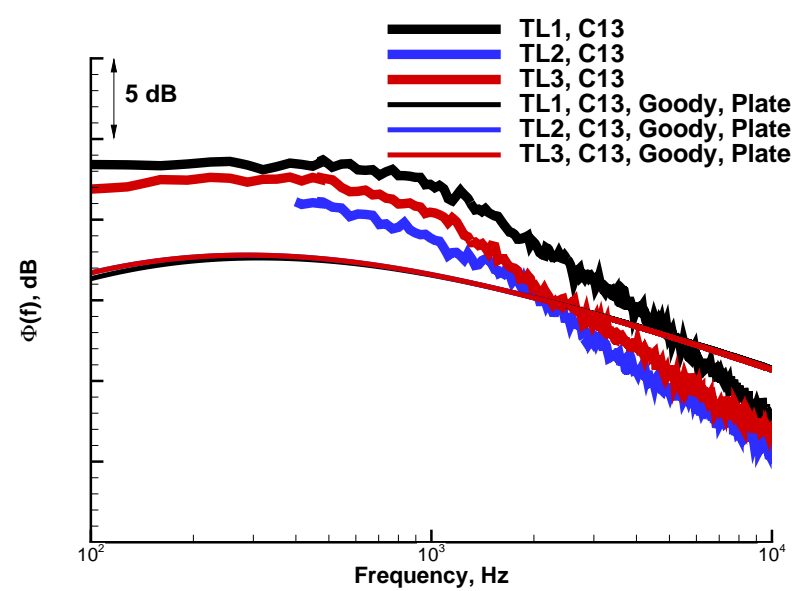

(a) Microphone data, zone one

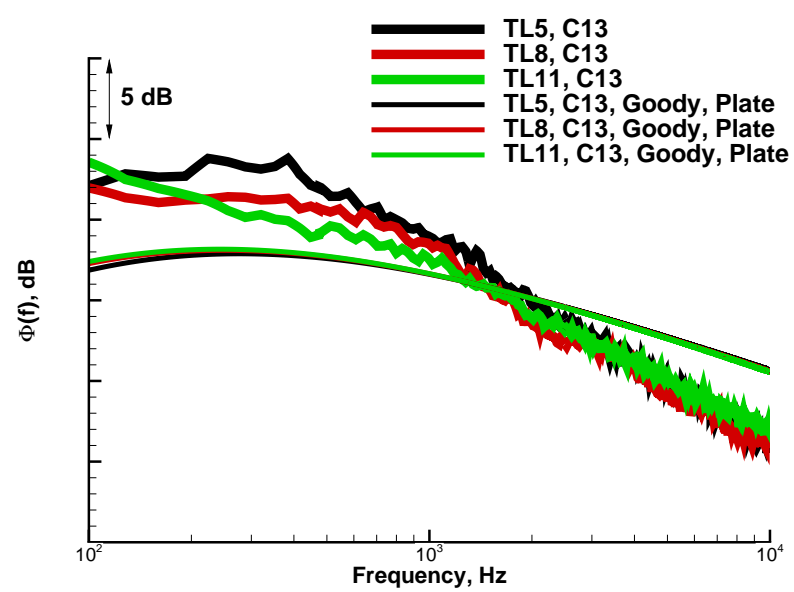

(b) Microphone data, zone two

Figure 17. Microphone measurement data compared to semi-empirical Goody model based on flat plate estimates

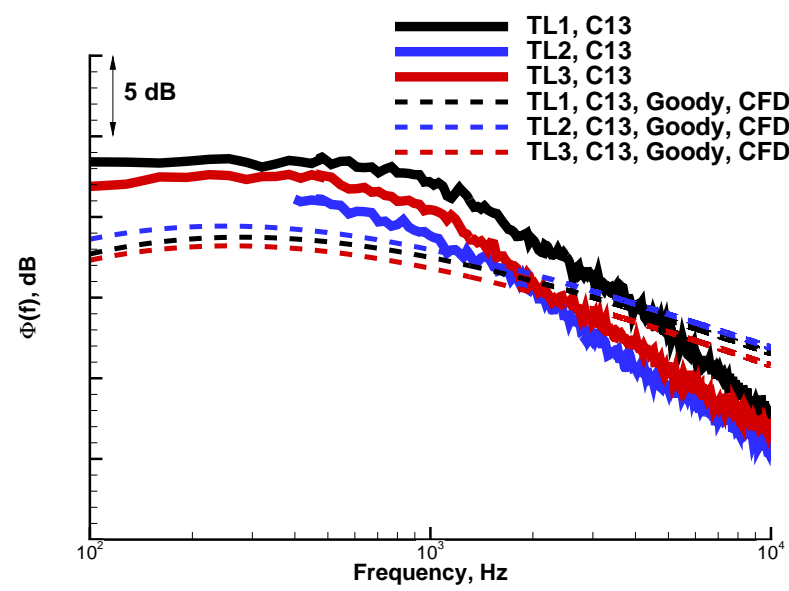

(a) Microphone data, zone one

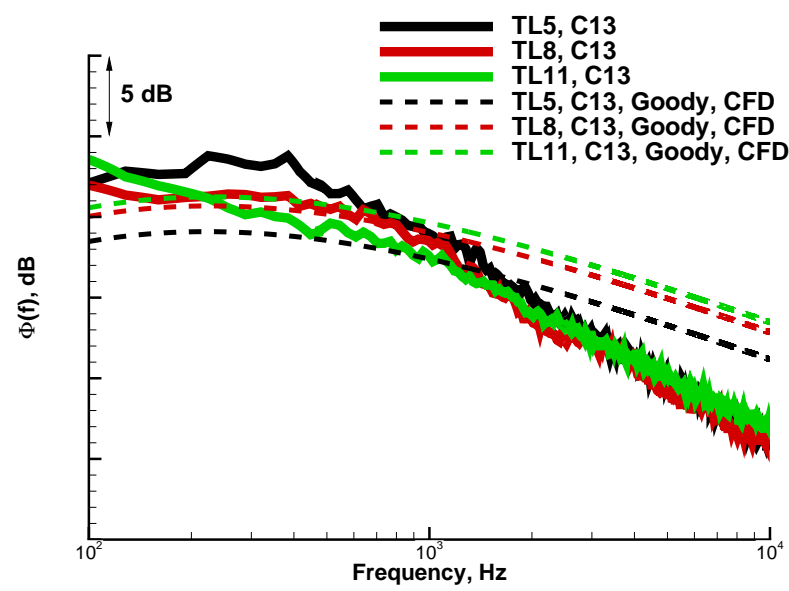

(b) Microphone data, zone two

Figure 18. Microphone measurement data compared to semi-empirical Goody model based on CFD

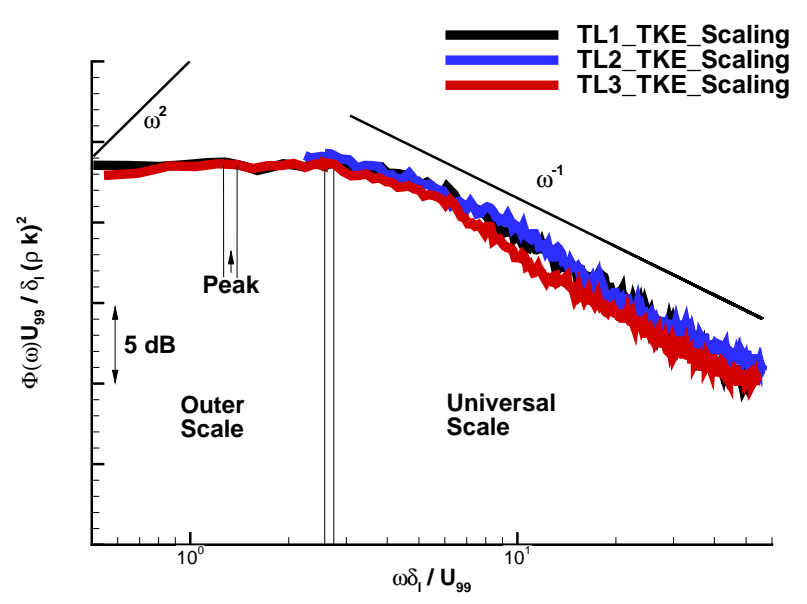

(a) Microphone data scaled, zone one

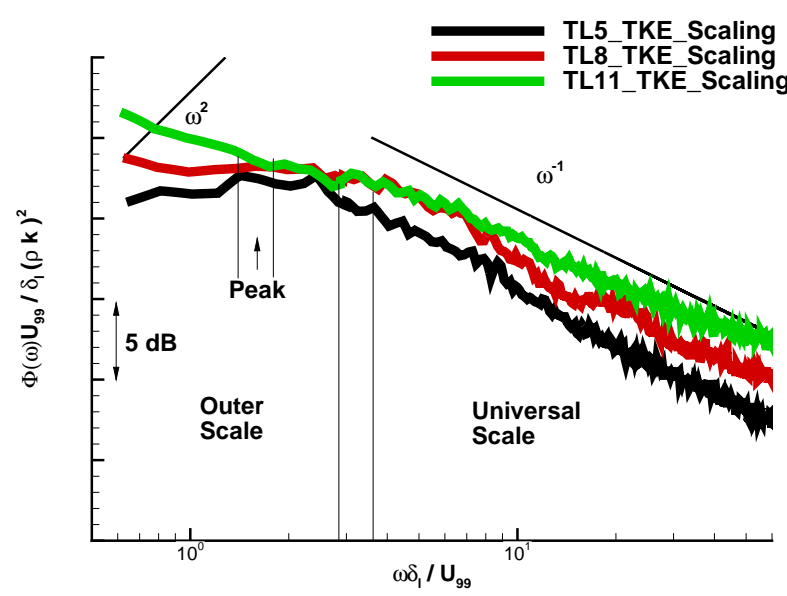

(b) Microphone data scaled, zone two

Figure 19. Microphone measurement data scaled with $k_{\max }$ 


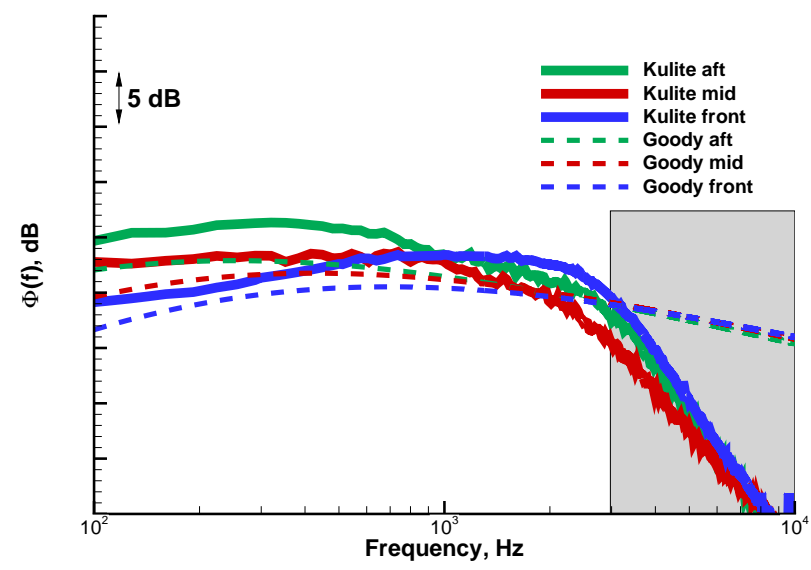

(a) Kulite data vs. Goody flat plate estimates

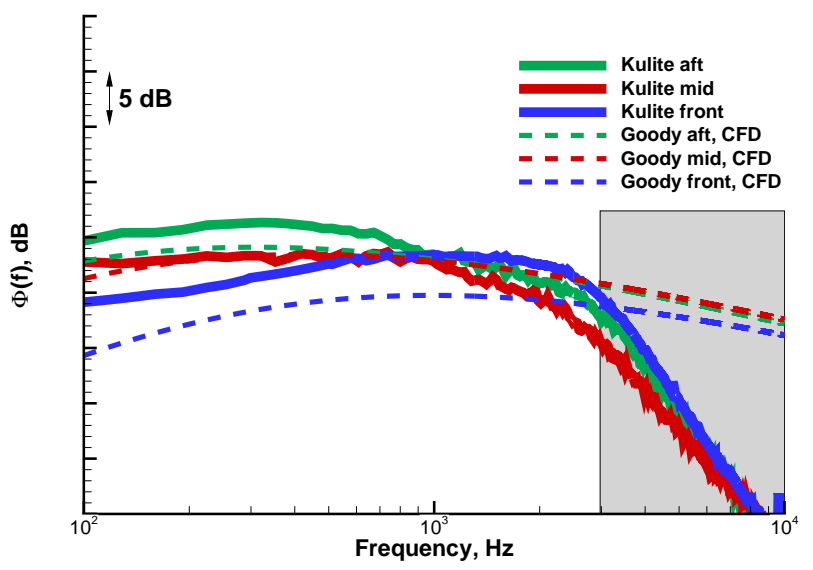

(b) Kulite data vs. Goody CFD

Figure 20. Kulite measurement data compared to semi-empirical models

CFD data) is presented in comparison with the measurement data. One can see that the semi-empirical models does not reproduce the measurement results either. However, differences in the predicted auto-spectra are obvious, which is an improvement compared with the prior prediction without CFD data. Taking further the analysed data from turbulence kinetic energy into account (fig. 15(a), 15(c), 15(e)), this could be one of the missing parameters in the auto-spectra prediction. Focusing e.g. on the prediction for TL1 in figure 18(a), the difference between measurement and prediction is about $6 \mathrm{~dB}$ at $f \approx 350 \mathrm{~Hz}$. The turbulence kinetic energy plot (figure 15(a)) shows a high energy value above the sensor and thus could improve the prediction result when taken into account. Beside this, the prediction for TL5 (fig. 18(b)) shows a similar behaviour, again a difference of $5 \mathrm{~dB}$ is present at $f \approx 350 \mathrm{~Hz}$ in comparison to the measurement. In this case, the amount of kinetic energy is not that strong than in the before mentioned case, but a peak value of energy exists above the sensor (fig. 15(e)). Furthermore, figure 15(f) shows decelerated flow over TL5, which could, in combination with the kinetic energy distribution explain the differences in the spectra. Concluding, it might be important to adapt the auto-spectra model with $k$ and $c_{p}$ distribution information, to predict measured auto-spectra correctly in shape and level.

Therefore, the model developed by CATLETT, ${ }^{25}$ which is a model for predicting auto-spectra under adverse pressure gradients, was tested. The prediction results were not able to fit the measurement data and does not constitute an improvement of the GoODY prediction results at the beforehand mentioned positions on the fuselage at cruise flight condition. The importance of $k$ as a parameter in the prediction of auto-spectra is illustrated by the results in figures 19(a) and 19(b). The absolute level of the spectra are scaled with $U_{e} / \delta_{99}(\rho k)^{2}$, where $U_{e}$ represents the edge velocity at $\delta_{99}, \rho$ is the density of the fluid and $k$ is the maximum value of the turbulence kinetic energy above the sensor position. The boundary layer thickness $\delta_{99}$ is defined by the before introduced pressure criterion, which is $99 \%$ of the far field total pressure. Furthermore, a Strouhal scaling is applied for the frequency axis, with $S h=\omega \delta_{99} / U_{e}$. Summing up, a good collapse of a large frequency range is reached and especially the peak values of the different auto-spectra show a very good collapse. Beside this, the classical slopes for the outer scaling and universal scaling region as well as the peak value location estimates are plotted in figures 19 (a) and 19(b), according to HwANG. ${ }^{11}$ Peak values are located between $\mathrm{Sh}=1.2 \ldots 1.8$ for the six different locations, which is based on the assumption that peaks are located at $\mathrm{Sh} \approx 50$ for $\mathrm{Sh}=\omega \delta_{l} / U_{\tau} \cdot{ }^{11}$ It can also be seen that the slope of $\omega^{-1}$ in the universal scaling range is found in the data. The $\omega^{2}$ slope at very low frequencies cannot be found in the data.

Beside this, one can see measured Kulite results in figures 20(a) and 20(b), with the Goody prediction based on flat plate estimates and CFD data, respectively. Again, results from the front, mid and aft section are shown, all data is recorded at the $15^{\circ}$ azimuthal rotated plane. Kulite results from the front are recorded at $\mathrm{x}=9.2 \mathrm{~m}$, in an area of accelerated flow compared to the free stream velocity. Furthermore, a positive pressure gradient was observed and denotes a deceleration in that area. The mid results are measured at $\mathrm{x}=15 \mathrm{~m}$, where $c_{p} \approx 0$ and no significant pressure gradient is present. Finally, the aft section Kulites were positioned at $\mathrm{x}=26 \mathrm{~m}$, which is again an area without pressure gradient and changed flow in terms 
of acceleration or deceleration. With respect to CFD data, it should be mentioned that the flow direction at the front position is twisted of $\vartheta_{C F D} \approx 3^{\circ}$ to the $\mathrm{x}$-axis, which is nearly the angle of attack $\left(\alpha=2^{\circ}\right)$. At the mid and aft section, the flow is twisted of $\vartheta_{C F D} \approx 12^{\circ}$ and $\vartheta_{C F D} \approx-5^{\circ}$, respectively, which also could have an influence on the spectra due to the curvature of the fuselage together with the turbulence kinetic energy distribution.

The Goody models in figures 20(a) and 20(b) can reproduce the spectral shape, in the confidence range, and the absolute levels in a good quality up to $3 \mathrm{kHz}$. Here, the CFD based Goody model (fig. 20(b)) constitutes an improvement of the prediction for the mid and aft section. The front section results are not reproduced in that quality, here the flat plate estimate based model seems to give better results.

\section{Conclusion}

A survey of the available semi-empirical models for the prediction of surface pressure auto-spectra was given. Beside this, an analysis of in-flight measured auto-spectra was done for several configurations at various positions with different sensors on the DLR Advanced Technology Research Aircraft (ATRA) Airbus A320. Comparison of the measurement data and the semi-empirical auto-spectra show that differences between prediction and measurement are existent. The amount of discrepancy in the data depends on the measurement position on the fuselage.

Computational fluid dynamic (CFD) calculations were performed for the ATRA at cruise flight conditions, to achieve detailed knowledge about the turbulent boundary layer (TBL) all over the fuselage. This data, in detail the boundary layer thickness $\left(\delta_{l}\right)$, pressure coefficient $\left(c_{p}\right)$, turbulence kinetic energy $(k)$, local flow direction, etc. were extracted and are used for the prediction of the semi-empirical auto-spectra models. This changeover in input data, from flat plate estimates to CFD based parameters shows an improvement of the prediction. Here, the Goody model, which was developed for low speed and incompressible flows, therefore not covering compressibility effects, yielded best approximations of the measured data at high speed flow. Therefore, the Goody model was selected as a starting point for further model development. Discrepancy between measurement and prediction is mostly evident in regions of high $k$ values and under pressure gradients. Data analysis showed that the maximum $k$ value above a sensor position can be used as a scaling parameter to normalise the auto-spectra peaks. This awareness shows that the turbulence history has a main impact on the prediction of auto-spectra, which is currently not implemented in the available models. The turbulence kinetic energy as well as the pressure gradient are supposed to be very important in further prediction of auto-spectra under turbulent flows on aircraft. This analysis shows that a CFD/CAA based prediction approach is necessary.

\section{Outlook}

It is planned to do further CFD analysis with an improved aircraft model and higher quality turbulence models for a more precise prediction of local parameters like the TBL edge velocity $U_{e}$ and thickness $\delta_{l}$. Improved CFD data should then be used to extend semi-empirical auto-spectra models with the ability to account for e.g. $k$. Furthermore, it is planned to develop a CFD/CAA based method for a generally applicable prediction of auto-spectra all over an aircraft fuselage. Beside this, it is intended to work on wavenumber-frequency spectra to predict the excitation including the space and time development of the TBL, again supported by numerical methods.

\section{Acknowledgments}

The authors would like to thank Airbus Operations GmbH (EPA5) for the financial support of this project.

\section{References}

${ }^{1}$ Miller, T., Gallman, J., and Moeller, M., "Review of Turbulent Boundary Layer Models for Acoustic Analysis," AIAA paper 2011-1083, January 2011.

${ }^{2}$ Spehr, C., Hennings, H., Bouhaj, M., Buchholz, H., Haxter, S., and Hebler, A., "In-flight Sound Measurements: A First Overview," AIAA paper 2012-2208, June 2012. 
${ }^{3}$ Haxter, S. and Spehr, C., "Two-Dimensional Evaluation of Turbulent Boundary Layer Pressure Fluctuations at Cruise Flight Conditions," AIAA paper 2012-2139, June 2012.

${ }^{4}$ Haxter, S. and Spehr, C., "Examination of the Influence of Flight Altitude and Speed on the Efimtsov Model Parameters," AIAA paper 2013-2028, May 2013.

${ }^{5}$ Haxter, S. and Spehr, C., "Listening to Turbulence: Measuring Coherence Decay at Different Positions on an Aircraft at Cruise Flight," AIAA paper 2014-3064, June 2014.

${ }^{6} \mathrm{Hu}, \mathrm{N}$., "Contributions of Different Aeroacoustic Sources to Aircraft Cabin Noise," AIAA paper 2013-2030, May 2013.

${ }^{7} \mathrm{Hu}$, N., Buchholz, H., Herr, M., Spehr, C., and Haxter, S., "Cabin Noise Source Ranking based on A320 Flight Test Data (Section 6.1), Aeroacoustics Research in Europe: The CEAS-ASC Report on 2012 Highlights," Journal of Sound and Vibration.

${ }^{8}$ Lowson, M., "Prediction of Boundary Layer Pressure Fluctuations," Technical Report AFFDL-TR 67-167s, U.S. Air Force Flight Dynamics Laboratory, Wright-Patterson Air Force Base, Ohio, 1968.

${ }^{9}$ Robertson, J., "Prediction of In-flight Fluctuating Pressure Environments Including Protuberance Induced Flow," CR119947, NASA, Wyle Laboratories, Huntsville Facility, 1971.

${ }^{10}$ Cockburn, J. and Robertson, J., "Vibration response of spacecraft shrouds to in-flight fluctuating pressures," Journal of Sound and Vibration, Vol. 33, No. 4, 1974, pp. 399-425.

${ }^{11}$ Hwang, Y., "Comparison of semi-empirical models for turbulent boundary layer wall pressure spectra," Journal of Sound and Vibration, Vol. 319, 2008, pp. 199-217.

${ }^{12}$ Chase, D., "Modeling the wavevector-frequency spectrum of turbulent boundary layer wall pressure," Journal of Sound and Vibration, Vol. 70, No. 1, 1980, pp. 29-67.

${ }^{13}$ Chase, D., "The character of the turbulent wall pressure spectrum at subconvective wavenumbers and a suggested comprehensive model," Journal of Sound and Vibration, Vol. 112, No. 1, 1987, pp. 125-147.

${ }^{14}$ Klabes, A., "Validation of a simulation model for the "Statistic Energy Analysis" (SEA) of the Airbus A320 for different flight parameters," internal report, Airbus Operations GmbH \& German Aerospace Center, 2012

${ }^{15}$ Efimtsov, B., "Characteristics of the field of turbulent wall pressure fluctuations at large Reynolds numbers," Soviet Physics - Acoustics, 1982.

${ }^{16}$ Efimtsov, B., "Similarity criteria for the spectra of wall pressure fluctuations in a turbulent boundary layer," Soviet Physics - Acoustics, 1984.

${ }^{17}$ Rackl, R. and Weston, A., "Modeling of Turbulent Boundary Layer Surface Pressure Fluctuation Auto and Cross SpectraVerification and Adjustments Based on TU-144LL Data," tech. rep. CR-2005-213938, NASA/The Boeing Company, Washington/Seattle, 2005.

${ }^{18}$ Howe, M., Acoustics of Fluid-Structure Interactions, Cambridge University Press, 2008.

${ }^{19}$ Goody, M., "Empirical Spectral Model of Surface Pressure Fluctuations," AIAA Journal, Vol. 42, No. 9, 2004.

${ }^{20}$ Smol'yakov, A., "Calculation of the Spectra of Pseudosound Wall-Pressure Fluctuations in Turbulent Boundary Layers," Acoustical Physics, Vol. 46, No. 3, 2000, pp. 342-347.

${ }^{21}$ Goody, M., An Experimental Investigation of Pressure Fluctuations in Three-Dimensional Turbulent Boundary Layers, Ph.D. thesis, Virginia Polytechnic Inst. and State Univ., Blacksburg, VA, Sept. 1999.

${ }^{22}$ DLR, "Technical Documentation of the DLR TAU-Code," Release 2014.1.0, German Aerospace Center, Institute of Aerodynamics and Flow Technology, Braunschweig, 2014.

${ }^{23}$ Eisfeld, B., "Implementation and validation of the Hellsten k-omega EARSM," tech. rep. IB 124-2003/33, German Aerospace Center, Institute of Aerodynamics and Flow Technology, 2003.

${ }^{24}$ Schlichting, H., Grenzschicht-Theorie, Verlag G. Braun, Karlsruhe, 1964.

${ }^{25}$ Catlett, M.R.; Forest, J.B.; Anderson, J.M.; Stewart, D.O., "Empirical Spectral Model of Surface Pressure Fluctuations beneath Adverse Pressure Gradients," AIAA paper 2014-2910, June 2014. 